MATHEMATICS OF COMPUTATION

Volume 73 , Number 246, Pages 541-567

S 0025-5718(03)01588-6

Article electronically published on July 28, 2003

\title{
ANALYSIS OF A FULLY DISCRETE FINITE ELEMENT METHOD FOR THE PHASE FIELD MODEL AND APPROXIMATION OF ITS SHARP INTERFACE LIMITS
}

\author{
XIAOBING FENG AND ANDREAS PROHL
}

\begin{abstract}
We propose and analyze a fully discrete finite element scheme for the phase field model describing the solidification process in materials science. The primary goal of this paper is to establish some useful a priori error estimates for the proposed numerical method, in particular, by focusing on the dependence of the error bounds on the parameter $\varepsilon$, known as the measure of the interface thickness. Optimal order error bounds are shown for the fully discrete scheme under some reasonable constraints on the mesh size $h$ and the time step size $k$. In particular, it is shown that all error bounds depend on $\frac{1}{\varepsilon}$ only in some lower polynomial order for small $\varepsilon$. The cruxes of the analysis are to establish stability estimates for the discrete solutions, to use a spectrum estimate result of Chen, and to establish a discrete counterpart of it for a linearized phase field operator to handle the nonlinear effect. Finally, as a nontrivial byproduct, the error estimates are used to establish convergence of the solution of the fully discrete scheme to solutions of the sharp interface limits of the phase field model under different scaling in its coefficients. The sharp interface limits include the classical Stefan problem, the generalized Stefan problems with surface tension and surface kinetics, the motion by mean curvature flow, and the Hele-Shaw model.
\end{abstract}

\section{INTRODUCTION}

In this paper we shall propose and analyze a fully discrete finite element timesplitting method for the phase field model

$$
\begin{aligned}
\varepsilon \alpha(\varepsilon) \varphi_{t}-\varepsilon \Delta \varphi+\frac{1}{\varepsilon} f(\varphi) & =s(\varepsilon) u & & \text { in } \Omega_{T}:=\Omega \times(0, T), \\
c(\varepsilon) u_{t}-\Delta u & =-\varphi_{t} & & \text { in } \Omega_{T}, \\
\frac{\partial u}{\partial n}=\frac{\partial \varphi}{\partial n} & =0 & & \text { in } \partial \Omega_{T}:=\partial \Omega \times(0, T), \\
\varphi=\varphi_{0}^{\varepsilon}, \quad u & =u_{0}^{\varepsilon} & & \text { in } \Omega \times\{0\},
\end{aligned}
$$

where $\Omega \subset \mathbf{R}^{N}(N=2,3)$ is a bounded domain with the smooth boundary $\partial \Omega$; $\alpha(\varepsilon), s(\varepsilon)$ and $c(\varepsilon)$ are nonnegative functions in $\varepsilon ; \varphi_{0}^{\varepsilon}$ and $u_{0}^{\varepsilon}$ are initial values which will be specified later in Section $[2$ and $f$ is the derivative of a smooth double equal

Received by the editor November 16, 2001 and, in revised form, October 30, 2002.

2000 Mathematics Subject Classification. Primary 65M60, 65M12, 65M15, 35B25, 35K57, 35Q99.

Key words and phrases. Phase field model, Allen-Cahn equation, Cahn-Hilliard equation, Stefan problem, motion by mean curvature, Hele-Shaw model, fully discrete finite element method. 
well potential taking its global minimum value 0 at $\varphi= \pm 1$. A typical example of $f$ is

$$
f(\varphi):=F^{\prime}(\varphi) \quad \text { and } \quad F(\varphi)=\frac{1}{4}\left(\varphi^{2}-1\right)^{2} .
$$

The existence of bistable states suggests that nonconvex energy is associated with the model (see the discussion below). We like to remark that nonsmooth potentials have also been considered in the literature for the phase field model; for that we refer to [7] and the references therein. We also note that the super-index $\varepsilon$ on the solution $\left(u^{\varepsilon}, \varphi^{\varepsilon}\right)$ is suppressed for notational brevity.

The phase field model for solidification was introduced by Caginalp [9], Collins and Levine [19], Fix [28] and Langer [30] to treat phenomena such as crystal growth and the fusion and joining of materials, which are not captured by the classical Stefan problem. The model consists of a heat equation (1.2) and a GinzburgLandau/Allen-Cahn equation (1.1) (cf. 4, 22)). Note that the original phase field model consists of equations (1.1) and (1.2), with $\alpha(\varepsilon)=O(1), s(\varepsilon)=O(1)$ and $c(\varepsilon)=O(1)$. In the model, $u$ represents the temperature and $\varphi$ is an order parameter which will vary continuously but somehow describes the phase of the material; $\varphi$ is scaled so that $\varphi \approx 1$ represents the liquid phase and $\varphi \approx-1$ represents the solid phase; and $\alpha, \varepsilon, s$ and $c$ are, respectively, the relaxation time, a microscopic scale, a surface tension scale, and the specific heat. We emphasize that the parameter $\varepsilon$ is usually small compared to the characteristic dimensions on the laboratory scale. The two boundary conditions in (1.3), the outward normal derivatives of $u$ and $\varphi$, vanish on $\partial \Omega$ and imply no gain or loss of heat energy through the walls of the container $\Omega$. For more physical background, derivation, and discussion of the phase field model and related equations, we refer to [2, 5, 6, 9, 19, 27, 28, 30, 32, 34] and the references therein.

It is known [27] that the phase field model can be formulated as a gradient flow with the Liapunov energy functional

$$
\mathcal{J}_{\varepsilon}(\varphi, u):=\int_{\Omega} \phi_{\varepsilon}(\varphi, u) \mathrm{d} x
$$

where

$$
\phi_{\varepsilon}(\varphi, u):=\frac{1}{2 \alpha(\varepsilon)}|\nabla \varphi|^{2}+\frac{1}{\varepsilon^{2} \alpha(\varepsilon)} F(\varphi)+\frac{c(\varepsilon) s(\varepsilon)}{2 \varepsilon \alpha(\varepsilon)} u^{2}
$$

in the Hilbert space $H_{0}^{-1} \times L^{2}$, where $H_{0}^{-1}$ denotes the mean-zero subspace of $H^{-1}$, the dual of the Sobolev space $H^{1}$. Note that the energy density $\phi_{\varepsilon}(\varphi, u)$ is not convex in $\varphi$.

In addition to the reason that the phase field model for solidification is widely accepted as a good model for treating phenomena which are not covered by the classical Stefan problem, it has also been used as a (computational) model to compute a wide range of sharp interface problems, including the classical and generalized Stefan problems, the motion by mean curvature flow and the Hele-Shaw model by taking advantage of the fact that the solution of the phase field model exists at all times and the singularities of the free boundaries do not pose either numerical or theoretical difficulties. Furthermore, it could provide sufficient information for the possible extensions of these free boundary problems beyond any singularities. Indeed, the connection between the phase field model and the sharp interface problems has been an extensively studied topic in recent years (cf. [2, 3, 7, 10, 11, 16, 22, 36, 37] and the references therein). It was first formally shown by Caginalp [10] that, as 
$\varepsilon \searrow 0$, the function $u$ tends to a limit $u^{0}$, which, together with a free boundary $\Gamma:=\bigcup_{0 \leq t \leq T}\left(\Gamma_{t} \times\{t\}\right)$, satisfies the following free boundary problem:

$$
\begin{aligned}
c^{0} u_{t}^{0}-\Delta u^{0} & =0 & & \text { in } \Omega_{T} \backslash \Gamma, \\
\frac{\partial u^{0}}{\partial n} & =0 & & \text { on } \partial \Omega_{T}, \\
V & =\frac{1}{2}\left[\frac{\partial u^{0}}{\partial n}\right]_{\Gamma} & & \text { on } \Gamma, \\
u^{0} & =-d^{0}\left(\kappa_{\Gamma}-\alpha^{0} V\right) & & \text { on } \Gamma, \\
u^{0} & =u_{0}^{0} & & \text { in } \Omega \times\{0\}, \\
\Gamma_{0} & =\Gamma_{00} & & \text { when } t=0,
\end{aligned}
$$

where $c^{0}, \alpha^{0}, c^{0}$ are nonnegative constants independent of $\varepsilon, V$ is the normal velocity of the interface $\Gamma$ (positive when the motion is directed toward the liquid), $\kappa_{\Gamma}$ is the sum of the principal curvatures of the interface (in space), $n$ is the unit outward normal to either $\partial \Omega$ or $\Gamma,\left[\frac{\partial u^{0}}{\partial n}\right]_{\Gamma}:=\frac{\partial u_{+}^{0}}{\partial n}-\frac{\partial u_{-}^{0}}{\partial n}$ denotes the jump of the normal derivatives of $u^{0}$ across $\Gamma$. Also $\varphi \rightarrow \pm 1$ uniformly in every compact subset of $\Omega_{T} \backslash \Gamma$ as $\varepsilon \searrow 0$. Later, the rigorous justification of this limit was successfully carried out by Caginalp and Chen [11], using a similar methodology to that in [3], under the assumption that the above free boundary problem has a unique classical solution. Also, Soner [36] proved the weak convergence of solutions of a phase field model with $\varphi$-dependent latent heat to the sharp interface limit in a very general setting that is applicable even when the sharp interface problem does not have a classical solution.

We note that when $s(\varepsilon)=0$ and $\alpha(\varepsilon)=1$, equation (1.1) decouples from (1.2) and becomes the Allen-Cahn equation 4

$$
\varphi_{t}-\Delta \varphi+\frac{1}{\varepsilon^{2}} f(\varphi)=0 .
$$

Its connection to the motion by mean curvature flow was established by de Mottoni and Schatzman [20] and to the generalized motion by mean curvature flow by Evans, Soner and Souganidis 22.

When $\alpha(\varepsilon)=c(\varepsilon)=0$ and $s(\varepsilon)=1$, equations (1.1) and (1.2) reduce to the Cahn-Hilliard equation [14]

$$
\varphi_{t}+\Delta\left(\varepsilon \Delta \varphi-\frac{1}{\varepsilon} f(\varphi)\right)=0
$$

The convergence of the Cahn-Hilliard equation to the Hele-Shaw model [33] was recently carried out by Alikakos, Bates and Chen [3].

It is clear that the study of the phase field model (1.1)-(1.4) is of great value for understanding solidification processes, in particular, in the presence of surface tension and surface kinetics, and for computing a wide range of sharp interface problems by taking advantage of the fact that the solution of the phase field model is known to exist for all time (cf. [21]). As pointed out earlier, this is particularly attractive from the computational point of view. The primary numerical challenge for solving the phase field model results from the presence of the $\varepsilon$-dependent coefficients in the equations of the model. Recall that the phase field model approximates the free boundary problem only when $\varepsilon$ becomes very small. On the other hand, the equations of the model become singularly perturbed heat equations for small $\varepsilon$. 
To resolve the solution numerically, one has to use small (space) mesh size $h$ and (time) step size $k$, which must be related to the parameter $\varepsilon$.

In the past fifteen years, numerical approximations of the phase field model with a fixed $\varepsilon$ have been developed and analyzed by several authors. Caginalp and Lin [12] and Lin [31] (also see 29]) proposed an explicit finite difference scheme and an implicit Crank-Nicolson scheme for the original phase field model, the convergence and error estimates of the schemes were shown under a restriction which is equivalent to $\alpha(\varepsilon) \leq c(\varepsilon)$. The restriction excludes some physically interesting cases. Chen and Hoffmann [17] proposed a fully discrete finite element method which uses the $P_{1}$ conforming finite element for space discretization and the backward Euler method for time discretization. An optimal order error estimate in $L^{\infty}\left(J ; L^{2}\right)$ was proved for the fully discrete method. A similar fully discrete finite element method was analyzed later by Yue 38 for the case of nonsmooth initial data. Caginalp and Socolovsky [13] proposed a computational method which consists of smoothing a sharp interface problem within the scaling of the distinguished limits of the phase field model that preserve physically important parameters. The computations from single-needle dendritic to faceted crystals are carried out continuously by adjusting the parameters in the method. Recently, Provatas, Goldenfeld and Dantzig 35] proposed an adaptive finite element algorithm using dynamic data structures, which enables us to simulate system sizes corresponding to experimental conditions.

We emphasize that the numerical analyses of all papers cited above were developed for the phase field model with a fixed $\varepsilon$. No special effort and attention were given to rigorously address issues such as how the mesh sizes $h$ and $k$ depend on $\varepsilon$ and how the error bounds depend on $\varepsilon$. In fact, since those error estimate results were derived using a Gronwall inequality type argument at the end of the derivations (cf. [17]), it is not hard to check that all error bounds contain a factor $\exp \left(\frac{T}{\varepsilon^{2}}\right)$, which clearly is not very useful when $\varepsilon$ is small.

Unlike the numerical works mentioned above, the focus of this paper is on approximating the solution of the phase field model (1.1)-(1.4) for small $\varepsilon$, which is the case for the applications we are interested in. The two primary goals of the paper are: (i) to analyze a fully discrete finite element method for the initial-boundary value problem (1.1)-(1.4) and to establish useful error bounds, which show growth only in low polynomial orders of $\frac{1}{\varepsilon}$, for the proposed scheme under some reasonable constraints on mesh sizes $h$ and $k$; (ii) to establish the convergence of the fully discrete finite element solution to the solutions of the sharp interface problems which are the distinguished limits of the phase field model under different scaling in its coefficients. To our knowledge, such error estimates and convergence results for the phase field model have not been known in the literature.

We remark that using a similar approach, parallel studies were carried out by the authors in 23] for the Allen-Cahn equation and the related curvature driven flows, and in 24, 25] for the Cahn-Hilliard equation and the Hele-Shaw problem. As pointed out earlier, the former corresponds to $s(\varepsilon)=0$ and $\alpha(\varepsilon)=1$ in the phase field model and the latter is obtained when $\alpha(\varepsilon)=c(\varepsilon)=0$ and $s(\varepsilon)=1$. The success of the approach, based on a spectrum estimate for the corresponding linearized operators, is due to the fact that it does not rely on the maximum and comparison principles, which are known not to hold for the Cahn-Hilliard equation and the phase field model. On the other hand, the required spectrum estimate does hold in each of the three cases, although the application of the estimate in the cases 
of the Cahn-Hilliard equation and the phase field model is rather delicate and complicated. In fact, the analysis of the phase field model (1.1)-(1.2) is more involved since the model consists of two nonstationary equations, with the second linked to the first via a time-derivative of $\varphi$; this coupling requires some new technique (e.g., use of the nonstandard test function in Step 2 of the proof of Theorem 3.1) in the error analysis. We note that such a technique is not needed in [23, 24, 25].

To avoid some complicated technicalities without compromising the main ideas and results, the analysis of this paper will be carried out for the case of the quartic potential given in (1.5), although the subsequent analysis and results apply to a general class of admissible double equal well potentials which satisfy some structural assumptions as described in [23, 24, 25].

The paper is organized as follows: In Section 2, we shall derive some a priori estimates for the solution of (1.1)-1.4. Special attention is given to the dependence of the solution on $\varepsilon$ in various norms. In Section 3 we propose and analyze a fully discrete (semi-implicit) finite element method (3.1)-(3.2) for the phase field model (1.1) - (1.4). The method consists of the backward Euler discretization in time and the $P_{1}$ conforming finite element discretization in space. Optimal error estimates in energy norm and a quasi-optimal error estimate in $\ell^{\infty}\left(J_{k} ; L^{\infty}\right)$ norm are obtained for the fully discrete solution in Theorems 3.1 and 3.2, where the error constants depend on $\frac{1}{\varepsilon}$ only in low polynomial orders. As in [23, 24, 25], the spectrum estimate for the linearized phase field operator (cf. Lemma 2.3) and its discrete counterpart (cf. Lemma 3.3) play a crucial role in the proofs. Finally, Section 4 is devoted to establishing the convergence of the fully discrete solution to the solution of the free boundary problem (1.7)-(1.12) in Theorems 4.2 and 4.3, which rely on the "polynomial growth-type" of error estimates with respect to $\varepsilon$. Combining the $\ell^{\infty}\left(J_{k} ; L^{\infty}\right)$ error estimates from Theorems 3.1 and 3.2 and the convergence result of [11], we show that the fully discrete numerical solution converges to the solution (including the free boundary) of the free boundary problem, provided that the latter admits a global (in time) classical solution.

This paper is a much condensed version of [26], where one can find more details, and a number of additional results as well as many helpful comments which could not be included here due to page limitation.

\section{Energy estimates for the Differential PROBlem}

In this section, we derive some energy estimates in various function spaces up to $H^{1}\left(J ; H^{2}(\Omega)\right)$ in terms of negative powers of $\varepsilon$ for the solution $(\varphi, u)$ to the phase field model (1.1)-(1.4) for given $\left(\varphi_{0}^{\varepsilon}, u_{0}^{\varepsilon}\right) \in\left[H^{2}(\Omega)\right]^{2}$, where $J=(0, T)$. Throughout this paper, the standard space, norm and inner product notation are adopted. Their definitions can be found in $[8,18$. In particular, $(\cdot, \cdot)$ denotes the standard inner product on $L^{2}(\Omega)$, and $H^{k}(\Omega)$ denotes the Sobolev space of the functions and their up-to- $k$ th order derivatives which are $L^{2}$-integrable. Also, $C$ and $\widetilde{C}$ are used to denote generic positive constants which are independent of $\varepsilon$ and the time and space mesh sizes $k$ and $h$.

In order to trace dependence of the solution on the small parameter $\varepsilon>0$, we assume that the initial functions $\varphi_{0}^{\varepsilon}$ and $u_{0}^{\varepsilon}$ satisfy the following assumption. 
General Assumption (GA)

There exist nonnegative $\varepsilon$-independent constants $\sigma_{j}$ for $j=1, \ldots, 4$ such that

$$
\begin{aligned}
\left|\varphi_{0}^{\varepsilon}\right| & \leq 1, \quad \text { in } \Omega \\
\mathcal{J}_{\varepsilon}\left(\varphi_{0}^{\varepsilon}, u_{0}^{\varepsilon}\right) & \leq C \varepsilon^{-2 \sigma_{1}} \\
\left\|\varepsilon \Delta \varphi_{0}^{\varepsilon}-\frac{1}{\varepsilon} f\left(\varphi_{0}^{\varepsilon}\right)+s(\varepsilon) u_{0}^{\varepsilon}\right\|_{L^{2}} & \leq C \varepsilon^{-\sigma_{2}}, \\
\left\|\nabla u_{0}^{\varepsilon}\right\|_{H^{\ell}} & \leq C \varepsilon^{-\sigma_{3+\ell}} \quad \text { for } \ell=0,1 .
\end{aligned}
$$

We now study the dependence of the solution of (1.1)-(1.4) on the given data of the problem, in particular on $\varepsilon$. The first lemma is a corollary of Theorem 3.1 of 11]. It shows boundedness of the first component $\varphi$ of the solution $(\varphi, u)$ of the phase field model, provided that the limiting free boundary problem (1.7)-(1.12) has a global (in time) classical solution.

Lemma 2.1. Let (2.1) hold. Suppose that the free boundary problem (1.7)-(1.12) has a unique global (in time) classical solution. Then there exists a family of smooth initial functions $\left\{\left(\varphi_{0}^{\varepsilon}, u_{0}^{\varepsilon}\right)\right\}_{0<\varepsilon \leq 1}$ and constants $\varepsilon_{0} \in(0,1]$ and $C_{0}>0$, such that for all $\varepsilon \in\left(0, \varepsilon_{0}\right)$ the solution $(\varphi, u)$ of the phase field model (1.1)-(1.4) with the above initial data satisfies

$$
\|\varphi\|_{L^{\infty}\left(\Omega_{T}\right)} \leq \frac{3}{2} C_{0} .
$$

Proof. Using a matched asymptotic expansion technique, it was shown in Section 4 of [11] that there exists a family of smooth approximate solutions $\left(\varphi_{A}^{\varepsilon}, u_{A}^{\varepsilon}\right)$ to the solution $(\varphi, u)$ of (1.1)-(1.4) satisfying the assumptions of Theorem 3.1 of [11]. One condition is $\left\|\varphi_{A}^{\varepsilon}\right\|_{L^{\infty}\left(\Omega_{T}\right)} \leq C_{0}$ for some $C_{0}>0$. It was then proved in Theorem 3.1 of [11] that $\left(\varphi_{A}^{\varepsilon}, u_{A}^{\varepsilon}\right)$ is very "close" to $(\varphi, u)$ in $L^{p}\left(\Omega_{T}\right)$ for some $p>2$ (see (3.3) on page 427 of [11]).

Now (2.5) follows from a regularization argument. The argument goes as follows in three steps: (i) modify $f$ into $\bar{f}$ such that $\bar{f}=f$ in $\left(-\frac{3}{2} C_{0}, \frac{3}{2} C_{0}\right)$ and $\bar{f}$ is linear for $|\varphi|>2 C_{0}$; (ii) it is not hard to show that the solution $(\bar{\varphi}, \bar{u})$ of the phase field model with the new nonlinearity $\bar{f}$ satisfies the estimate (2.5) when $\varepsilon \in\left(0, \varepsilon_{0}\right)$ for some small $\varepsilon_{0} \in(0,1]$; (iii) it follows from the uniqueness of the solution of the phase field model that $u \equiv \bar{u}$.

Since (1.1)-(1.4) is a gradient flow for the functional (1.6), it is easy to check that the model satisfies the following energy law:

$$
\frac{d}{d t} \mathcal{J}_{\varepsilon}(u(t))=-\left\|\phi_{t}\right\|_{L^{2}}^{2}-\frac{s(\varepsilon)}{\varepsilon \alpha(\varepsilon)}\|\nabla u\|_{L^{2}}^{2},
$$

where $\mathcal{J}_{\varepsilon}(\cdot, \cdot)$ is defined by (1.6). Based on this energy law and Lemma 2.1, repeatedly using the bootstrapping argument, we can show the following a priori estimates for the solution of (1.1)-(1.4). For the detailed derivation, see [26]. 
Lemma 2.2. Let $\left(\varphi_{0}^{\varepsilon}, u_{0}^{\varepsilon}\right)$ satisfy (GA), $f(\varphi)=\varphi^{3}-\varphi$, and let Lemma 2.1 hold. Then, the solution of (1.1)-(1.4) satisfies the following estimates:

$$
\begin{aligned}
& \operatorname{ess} \sup _{[0, \infty]}\left\{\frac{\varepsilon}{2}\|\nabla \varphi\|_{L^{2}}^{2}+\frac{1}{\varepsilon}\|F(\varphi)\|_{L^{1}}+\frac{s(\varepsilon) c(\varepsilon)}{2}\|u\|_{L^{2}}^{2}\right\} \\
& \quad+\int_{0}^{\infty}\left[\varepsilon \alpha(\varepsilon)\left\|\varphi_{t}(s)\right\|_{L^{2}}^{2}+\frac{s(\varepsilon)}{2}\|\nabla u(s)\|_{L^{2}}^{2}\right] \mathrm{d} s \leq \varepsilon \alpha(\varepsilon) \mathcal{J}_{\varepsilon}\left(\varphi_{0}^{\varepsilon}, u_{0}^{\varepsilon}\right),
\end{aligned}
$$

$$
\operatorname{ess} \sup _{[0, \infty]}\left\{c(\varepsilon)\|\nabla u\|_{L^{2}}^{2}\right\}+\int_{0}^{\infty}\left[\frac{c(\varepsilon)^{2}}{2}\left\|u_{t}(s)\right\|_{L^{2}}^{2}+\|\Delta u(s)\|_{L^{2}}^{2}\right] \mathrm{d} s
$$

$$
\leq \mathcal{J}_{\varepsilon}\left(\varphi_{0}^{\varepsilon}, u_{0}^{\varepsilon}\right)+C[1+c(\varepsilon)] \varepsilon^{-2 \sigma_{3}},
$$

$$
\begin{aligned}
& \int_{0}^{\infty}\|\Delta \varphi(s)\|_{L^{2}}^{2} \mathrm{~d} s \leq\left[\frac{\alpha(\varepsilon)}{\varepsilon^{2}}+\varepsilon s(\varepsilon) \alpha(\varepsilon)\right] \mathcal{J}_{\varepsilon}\left(\varphi_{0}^{\varepsilon}, u_{0}^{\varepsilon}\right), \\
& \text { ess } \sup _{[0, \infty]}\left\{\alpha(\varepsilon)\left\|\varphi_{t}\right\|_{L^{2}}^{2}\right\}+\int_{0}^{\infty}\left\|\nabla \varphi_{t}(s)\right\|_{L^{2}}^{2} \mathrm{~d} s \leq \mathcal{B}_{1},
\end{aligned}
$$

$$
\text { ess } \sup _{[0, \infty]}\|\Delta \varphi\|_{L^{2}}^{2} \leq \mathcal{B}_{2} \text {, }
$$

$$
\int_{0}^{\infty}\left\|\varphi_{t t}(s)\right\|_{H^{-1}}^{2} \mathrm{~d} s \leq \mathcal{B}_{3}
$$$$
\text { ess } \sup _{[0, \infty]}\left\{c(\varepsilon)\left\|u_{t}\right\|_{L^{2}}^{2}\right\}+\int_{0}^{\infty}\left\|\nabla u_{t}\right\|_{L^{2}}^{2} \mathrm{~d} s \leq \mathcal{B}_{4},
$$

(viii) $\quad$ ess $\sup _{[0, \infty]}\|\Delta u\|_{L^{2}}^{2} \leq \mathcal{B}_{5}$,

$$
\int_{0}^{\infty}\left\|u_{t t}\right\|_{H^{-1}}^{2} \mathrm{~d} s \leq \mathcal{B}_{6},
$$

where $\mathcal{B}_{i} \equiv \mathcal{B}_{i}(\varepsilon ; c(\varepsilon), s(\varepsilon), \alpha(\varepsilon))$ for $i=1, \ldots, 6$ are defined as follows:

$$
\begin{gathered}
\mathcal{B}_{1}:=\left[\frac{2}{\varepsilon^{2}}+\frac{s(\varepsilon)^{2}}{c(\varepsilon)^{2}}\right]\left(\mathcal{J}_{\varepsilon}\left(\varphi_{0}^{\varepsilon}, u_{0}^{\varepsilon}\right)+C \varepsilon^{-2 \sigma_{3}}\right)+\frac{C}{\alpha(\varepsilon)} \varepsilon^{-2\left(\sigma_{2}+1\right)}, \\
\mathcal{B}_{2}:=C\left[1+\frac{\alpha(\varepsilon) s(\varepsilon)^{2}}{c(\varepsilon)^{2}}+\frac{s(\varepsilon)^{2}}{c(\varepsilon)}+\frac{\alpha(\varepsilon)}{\varepsilon}\right] \varepsilon^{2 \min \left\{-\sigma_{2},-\sigma_{3}\right\}-2}, \\
\mathcal{B}_{3}:=\frac{\mathcal{B}_{1}}{\alpha(\varepsilon)^{2}}+\frac{s(\varepsilon)^{2}}{\varepsilon^{2} \alpha(\varepsilon)^{2} c(\varepsilon)^{2}}\left\{\mathcal{J}_{\varepsilon}\left(\varphi_{0}^{\varepsilon}, u_{0}^{\varepsilon}\right)+C[1+c(\varepsilon)] \varepsilon^{-2 \sigma_{3}}\right\} \\
\quad+\frac{1}{\varepsilon^{4} \alpha(\varepsilon)^{2}}\left\{\mathcal{J}_{\varepsilon}\left(\varphi_{0}^{\varepsilon}, u_{0}^{\varepsilon}\right)\right\}, \\
\mathcal{B}_{4}:=\mathcal{B}_{3}+\frac{\mathcal{J}_{\varepsilon}\left(\varphi_{0}^{\varepsilon}, u_{0}^{\varepsilon}\right)}{c(\varepsilon)^{2}}+\frac{C[1+c(\varepsilon)]}{c(\varepsilon)^{2}} \varepsilon^{-2 \sigma_{3}}+\frac{1}{2 c(\varepsilon)}\left[\varepsilon^{-2 \sigma_{4}}+\frac{\varepsilon^{-2\left(\sigma_{2}+1\right)}}{\alpha(\varepsilon)^{2}}\right], \\
\mathcal{B}_{5}:=c(\varepsilon) \mathcal{B}_{4}+\frac{\mathcal{B}_{1}}{\alpha(\varepsilon)}, \quad \mathcal{B}_{6}:=\frac{1}{c(\varepsilon)^{2}}\left[\mathcal{B}_{4}+\mathcal{B}_{3}\right] .
\end{gathered}
$$

In addition, if

$$
\lim _{s \rightarrow 0^{+}}\left\|\nabla \varphi_{t}(s)\right\|_{L^{2}} \leq C \varepsilon^{-\xi_{1}}, \quad \lim _{s \rightarrow 0^{+}}\left\|\nabla u_{t}(s)\right\|_{L^{2}} \leq C \varepsilon^{-\xi_{2}},
$$


for some $\xi_{1}, \xi_{2} \geq 0$, then it also holds that

$$
\begin{gathered}
\text { (x) } \int_{0}^{\infty}\left\|\varphi_{t t}(s)\right\|_{L^{2}}^{2} \mathrm{~d} s+\operatorname{ess} \sup _{[0, \infty]}\left\|\nabla \varphi_{t}\right\|_{L^{2}}^{2}+\frac{\varepsilon^{2}}{\alpha(\varepsilon)} \int_{0}^{\infty}\left\|\Delta \varphi_{t}(s)\right\|_{L^{2}}^{2} \mathrm{~d} s \leq \mathcal{B}_{7}, \\
\text { (xi) } \quad c(\varepsilon) \int_{0}^{\infty}\left\|u_{t t}(s)\right\|_{L^{2}}^{2} \mathrm{~d} s+\operatorname{ess} \sup _{[0, \infty]}\left\|\nabla u_{t}\right\|_{L^{2}}^{2} \\
+[1+c(\varepsilon)] \int_{0}^{\infty}\left\|\Delta u_{t}(s)\right\|_{L^{2}}^{2} \mathrm{~d} s \leq \mathcal{B}_{8}
\end{gathered}
$$

where

$$
\begin{aligned}
\mathcal{B}_{7} & :=\frac{C \mathcal{J}_{\varepsilon}\left(\varphi_{0}^{\varepsilon}, u_{0}^{\varepsilon}\right)}{\varepsilon^{4} \alpha(\varepsilon)}+\frac{s(\varepsilon)^{2}}{\varepsilon^{2} \alpha(\varepsilon) c(\varepsilon)^{2}}\left\{\mathcal{J}_{\varepsilon}\left(\varphi_{0}^{\varepsilon}, u_{0}^{\varepsilon}\right)+C[1+c(\varepsilon)] \varepsilon^{-2 \sigma_{3}}\right\}, \\
\mathcal{B}_{8} & :=\frac{1}{c(\varepsilon)} \mathcal{B}_{7}+\varepsilon^{-2 \xi_{2}} .
\end{aligned}
$$

We conclude this section by citing the following result of [15, 11] on a low bound estimate of the (generalized) spectrum of the linearized phase field operator $\mathcal{L}_{P F}$ with respect to the operator $\mathcal{N}_{P F}$,

$$
\mathcal{L}_{P F}:=\left(\begin{array}{cc}
-\varepsilon \Delta-\frac{1}{\varepsilon} f^{\prime}(\varphi) I & \Theta \\
I & -\Delta
\end{array}\right), \quad \mathcal{N}_{P F}:=\left(\begin{array}{cc}
\alpha(\varepsilon) \varepsilon I & -s(\varepsilon) I \\
\Theta & c(\varepsilon) I
\end{array}\right),
$$

where $I$ and $\Theta$ denote the identity and zero operators, and $\varphi$ is the solution of the phase field model (1.1)-(1.4).

This estimate plays a crucial role in our error analysis.

Lemma 2.3. Suppose the assumptions of Lemma 2.1 hold. Let $\lambda_{P F}$ denote the smallest (generalized) eigenvalue of the eigenvalue problem

$$
\mathcal{L}_{P F}\left(\begin{array}{c}
\psi \\
w
\end{array}\right)=\lambda \mathcal{N}_{P F}\left(\begin{array}{c}
\psi \\
w
\end{array}\right) .
$$

Then there exist $0<\varepsilon_{0}<<1$ and another postive, $\varepsilon$-independent constant $C_{0}$ such that $\lambda_{P F}$ satisfies $\lambda_{P F} \geq-C_{0}$ for $\varepsilon \in\left(0, \varepsilon_{0}\right)$. Equivalently,

$$
\lambda_{P F}=\inf _{\substack{\psi \in H^{1}(\Omega) \\ w \in H^{2}(\Omega)}} \frac{\varepsilon\|\nabla \psi\|_{L^{2}}^{2}+\varepsilon^{-1}\left(f^{\prime}(\varphi) \psi, \psi\right)+\frac{s(\varepsilon)}{c(\varepsilon)}\|\Delta w-\psi\|_{L^{2}}^{2}}{\varepsilon \alpha(\varepsilon)\|\psi\|_{L^{2}}^{2}+s(\varepsilon)\|\nabla w\|_{L^{2}}^{2}} \geq-C_{0},
$$

for $\varepsilon \in\left(0, \varepsilon_{0}\right)$, where $\varphi$ denotes the first component of the solution vector $(\varphi, u)$ to the phase field model (1.1)-(1.4).

Proof. The estimate (2.8) was established by X. Chen in [15] for any $\varphi$ which satisfies some special profile (cf. (1.10) on page 1374 and Theorem 1.1 on page 1375 of [15]). It was shown in [1] that the first component $\varphi$ of the solution vector $(\varphi, u)$ to the phase field model (1.1)-(1.4) indeed satisfies the required profile (cf. Theroem 3.1 of [11]) for sufficiently small $\varepsilon$. The conclusion of the lemma then follows from combining these two results.

Remark 2.1. Since the proof of (2.8) is based on the convergence result of [11, which says that the solution of the phase field model (1.1)-(1.4) for a certain class of initial conditions converges to the classical solution of the free boundary problem (1.7)(1.12) as $\varepsilon \rightarrow 0$, hence, the proof suggests that the validity of (2.8) also depends on the choice of the initial conditions. As far as we know, it is an open question 
whether estimate (2.8) holds for "general" initial data (see Remark 2.3 of [1] for more discussions). This is the reason why the subsequent main results of this paper are established under the same initial condition constraint.

\section{ERROR ANALYSIS FOR A FULLY DISCRETE FINITE ELEMENT APPROXIMATION}

Let $\mathcal{T}_{h}$ be a quasi-uniform triangulation of $\Omega$ such that $\bar{\Omega}=\bigcup_{K \in \mathcal{T}_{h}} \bar{K}\left(K \in \mathcal{T}_{h}\right.$ are tetrahedrons in the case $N=3)$. Here $h:=\max _{K \in \mathcal{T}_{h}} h_{K}$ denotes the mesh size of $\mathcal{T}_{h}$; see [8, 18] for further details. Let $V_{h}$ be the finite element subspace of $H^{1}(\Omega)$ associated with $\mathcal{T}_{h}$ and consisting of continuous and piecewise linear functions on $\mathcal{T}_{h}$, that is,

$$
V_{h}:=\left\{v_{h} \in C(\bar{\Omega}):\left.v_{h}\right|_{K} \in P_{1}(K), \forall K \in \mathcal{T}_{h}\right\} .
$$

In this section, we shall establish stability and convergence properties for the following fully discrete finite element scheme: Find $\left(\Phi^{m}, U^{m}\right) \in\left[V_{h}\right]^{2}$ for $m=1,2$, $\ldots, M$ satisfying

$$
\begin{gathered}
\varepsilon \alpha(\varepsilon)\left(d_{t} \Phi^{m}, \eta_{h}\right)+\varepsilon\left(\nabla \Phi^{m}, \nabla \eta_{h}\right)+\frac{1}{\varepsilon}\left(f\left(\Phi^{m}\right), \eta_{h}\right)=s(\varepsilon)\left(U^{m, \mu}, \eta_{h}\right), \\
c(\varepsilon)\left(d_{t} U^{m}, v_{h}\right)+\left(\nabla U^{m}, \nabla v_{h}\right)=-\left(d_{t} \Phi^{m}, v_{h}\right),
\end{gathered}
$$

for all $\left(\eta_{h}, v_{h}\right) \in\left[V_{h}\right]^{2}$, together with some starting value $\left(\Phi^{0}, U^{0}\right) \in\left[V_{h}\right]^{2}$, where $U^{m, \mu}:=\mu U^{m}+(1-\mu) U^{m-1}$ for $\mu=0,1$.

Due to page limitations, in this section we only consider the semi-implicit case $\mu=0$, and we refer to $[26$ for the analysis for the fully implicit case $\mu=1$. Clearly, when $\mu=0$, the iterates $\left(\Phi^{m}, U^{m}\right)$ are computed in succession at each iteration step, which allows for the immediate use of existing computer codes for solving the Allen-Cahn equation and the heat equation, respectively.

For the error analysis, we need to introduce the elliptic projection operator $P_{h}: H^{1}(\Omega) \rightarrow V_{h}$,

$$
\begin{aligned}
\left(\nabla\left[\psi-P_{h} \psi\right], \nabla v_{h}\right) & =0 \quad \forall v_{h} \in V_{h}, \\
\left(\psi-P_{h} \psi, 1\right) & =0 .
\end{aligned}
$$

It is well known that $P_{h}$ has the following approximation properties [8, 18]:

$$
\begin{aligned}
& \left\|\psi-P_{h} \psi\right\|_{L^{2}}+h\left\|\nabla\left(\psi-P_{h} \psi\right)\right\|_{L^{2}} \leq C h^{2}\|\psi\|_{H^{2}} \forall \psi \in H^{2}(\Omega), \\
& \left\|\psi-P_{h} \psi\right\|_{L^{\infty}} \leq C h^{\frac{4-N}{2}}|\ln h|^{\frac{3-N}{2}}\|\psi\|_{H^{2}} \quad \forall \psi \in H^{2}(\Omega), \\
& \left\|\left(\psi-P_{h} \psi\right)_{t}\right\|_{L^{2}\left(J ; L^{2}\right)} \leq C h^{2}\left\|\psi_{t}\right\|_{L^{2}\left(J ; H^{2}\right)} \quad \forall \psi \in H^{1}\left(J ; H^{2}\right), \\
& \left\|\left(\psi-P_{h} \psi\right)_{t}\right\|_{L^{2}\left(J ; H^{-1}\right)} \leq C h^{2}\|\psi\|_{*} \quad \forall \psi \in W,
\end{aligned}
$$

where

$$
\begin{aligned}
W & =\left\{w ; w \in H^{1}\left(J ; H^{1}\right),\|w\|_{*}<\infty\right\} \\
\|w\|_{*} & =\left(\|w\|_{H^{1}\left(J ; H^{1}\right)}^{2}+\sum_{i, j=1}^{N}\left\|\partial_{x_{j}} \partial_{x_{i}} w_{t}\right\|_{L^{2}\left(J ; H^{-1}\right)}^{2}\right)^{\frac{1}{2}} .
\end{aligned}
$$

We note that a short proof of (3.6) can be found in [23.

To be used in later analysis, we also define the discrete (negative) Laplace operator $-\Delta_{h}: V_{h} \cup H^{1}(\Omega) \longrightarrow V_{h}$ by

$$
\left(-\Delta_{h} \psi, \eta_{h}\right)=\left(\nabla \psi, \nabla \eta_{h}\right) \quad \forall \eta_{h} \in V_{h} \cap H^{1}(\Omega) .
$$

We now state a basic stability result for the fully discrete scheme. 
Lemma 3.1. Let $f(\varphi)=\varphi^{3}-\varphi$ and $c(\varepsilon) \geq c_{0}>0$. The solution $\left\{\left(\Phi^{m}, U^{m}\right)\right\}_{m=0}^{M}$ of (3.1)-3.2 satisfies for values $k<\min \left\{\alpha(\varepsilon)^{2} \varepsilon^{4}, \frac{c(\varepsilon)^{2}}{s(\varepsilon)^{2} \varepsilon^{2}}\right\}$,

$$
\begin{aligned}
& \max _{0 \leq m \leq M}\left\{\varepsilon\left\|\nabla \Phi^{m}\right\|_{L^{2}}^{2}+\frac{1}{\varepsilon}\left\|F\left(\Phi^{m}\right)\right\|_{L^{1}}+s(\varepsilon) c(\varepsilon)\left\|U^{m}\right\|_{L^{2}}^{2}\right\} \\
& \quad+k \sum_{m=0}^{M}\left\{\varepsilon\left(\alpha(\varepsilon)-\frac{\sqrt{k}}{\varepsilon^{2}}\right)\left\|d_{t} \Phi^{m}\right\|_{L^{2}}^{2}+\frac{s(\varepsilon)}{2}\left\|\nabla U^{m}\right\|_{L^{2}}^{2}+\frac{\varepsilon k}{2}\left\|\nabla d_{t} \Phi^{m}\right\|_{L^{2}}^{2}\right. \\
& \left.\quad+\frac{k}{2 \varepsilon}\left\|d_{t}\left(\left|\Phi^{m}\right|^{2}-1\right)\right\|_{L^{2}}^{2}+\frac{k s(\varepsilon)[c(\varepsilon)-\varepsilon s(\varepsilon) \sqrt{k}]}{2}\left\|d_{t} U^{m}\right\|_{L^{2}}^{2}\right\} \\
& \leq \varepsilon \alpha(\varepsilon) \mathcal{J}_{\varepsilon}\left(\varphi_{0}^{\varepsilon}, u_{0}^{\varepsilon}\right) .
\end{aligned}
$$

Proof. We first rewrite $f\left(\Phi^{m}\right)$ as follows:

$$
f\left(\Phi^{m}\right)=\frac{1}{2}\left(\left|\Phi^{m}\right|^{2}-1\right)\left(\left[\Phi^{m}+\Phi^{m-1}\right]+k d_{t} \Phi^{m}\right) .
$$

Multiplying the equation by $d_{t} \Phi^{m}$ gives

$$
\begin{aligned}
& \frac{1}{2 \varepsilon}\left(f\left(\Phi^{m}\right), d_{t} \Phi^{m}\right)=\frac{1}{2 \varepsilon}\left(\left|\Phi^{m}\right|^{2}-1, d_{t}\left(\left|\Phi^{m}\right|^{2}-1\right)\right) \\
& \quad+\frac{k}{2 \varepsilon}\left(\left|\Phi^{m}\right|^{2}-1,\left|d_{t} \Phi^{m}\right|^{2}\right) \\
& \geq \frac{1}{2 \varepsilon}\left(\left|\Phi^{m}\right|^{2}-1 \pm\left(\left|\Phi^{m-1}\right|^{2}-1\right), d_{t}\left(\left|\Phi^{m}\right|^{2}-1\right)\right)-\frac{k}{2 \varepsilon}\left\|d_{t} \Phi^{m}\right\|_{L^{2}}^{2} \\
& \geq \frac{1}{2 \varepsilon} d_{t}\left\|\left|\Phi^{m}\right|^{2}-1\right\|_{L^{2}}^{2}+\frac{k}{2 \varepsilon}\left\|d_{t}\left(\left|\Phi^{m}\right|^{2}-1\right)\right\|_{L^{2}}^{2}-\frac{k}{2 \varepsilon}\left\|d_{t} \Phi^{m}\right\|_{L^{2}}^{2} .
\end{aligned}
$$

We now test (3.1) with $d_{t} \Phi^{m}$ and (3.2) with $s(\varepsilon) U^{m}$. Adding the resulting equations leads to

$$
\begin{aligned}
\varepsilon \alpha(\varepsilon) & \left\|d_{t} \Phi^{m}\right\|_{L^{2}}^{2}+\frac{\varepsilon}{2} d_{t}\left\|\nabla \Phi^{m}\right\|_{L^{2}}^{2}+\frac{\varepsilon k}{2}\left\|\nabla d_{t} \Phi^{m}\right\|_{L^{2}}^{2} \\
& +\frac{1}{2 \varepsilon} d_{t}\left\|\left|\Phi^{m}\right|^{2}-1\right\|_{L^{2}}^{2}+\frac{k}{2 \varepsilon}\left\|d_{t}\left(\left|\Phi^{m}\right|^{2}-1\right)\right\|_{L^{2}}^{2} \\
& +\frac{c(\varepsilon) s(\varepsilon)}{2} d_{t}\left\|U^{m}\right\|_{L^{2}}^{2}+\frac{k c(\varepsilon) s(\varepsilon)}{2}\left\|d_{t} U^{m}\right\|_{L^{2}}^{2}+s(\varepsilon)\left\|\nabla U^{m}\right\|_{L^{2}}^{2} \\
\leq & \frac{k}{2 \varepsilon}\left\|d_{t} \Phi^{m}\right\|_{L^{2}}^{2}-s(\varepsilon) k\left(d_{t} U^{m}, d_{t} \Phi^{m}\right) \\
\leq & \frac{\sqrt{k}}{\varepsilon}\left\|d_{t} \Phi^{m}\right\|_{L^{2}}^{2}+\frac{k^{\frac{3}{2}} \varepsilon s(\varepsilon)^{2}}{2}\left\|d_{t} U^{m}\right\|_{L^{2}}^{2} .
\end{aligned}
$$

The assertion follows from summing (3.11) over $m$ from 0 to $M$.

Lemma 3.2. Let $f(\varphi)=\varphi^{3}-\varphi$. Under the mesh constraint of Lemma 3.1, the solution $\left\{U^{m}\right\}_{m=0}^{M}$ of (3.1)-(3.2) also satisfies the following stability estimate:

$$
\begin{gathered}
\max _{1 \leq m \leq M}\left\{\varepsilon \alpha(\varepsilon)\left\|d_{t} \Phi^{m}\right\|_{L^{2}}^{2}+s(\varepsilon)\left\|\nabla U^{m}\right\|_{L^{2}}^{2}\right\}+k \sum_{m=1}^{M}\left\{\frac{\varepsilon \alpha(\varepsilon) k}{8}\left\|d_{t}^{2} \Phi^{m}\right\|_{L^{2}}^{2}\right. \\
\left.+\varepsilon\left\|\nabla d_{t} \Phi^{m}\right\|_{L^{2}}^{2}+c(\varepsilon) s(\varepsilon)\left\|d_{t} U^{m}\right\|_{L^{2}}^{2}+\frac{s(\varepsilon) k}{2}\left\|\nabla d_{t} U^{m}\right\|_{L^{2}}^{2}\right\} \\
\leq C\left\{\frac{\alpha(\varepsilon)\left[1+s(\varepsilon)^{2} \varepsilon^{3}\right]}{\varepsilon} \mathcal{J}_{\varepsilon}\left(\varphi_{0}^{\varepsilon}, u_{0}^{\varepsilon}\right)+s(\varepsilon) \varepsilon^{-2 \sigma_{3}}\right\} .
\end{gathered}
$$


Proof. First, take $\eta_{h}=d_{t} \Phi^{m}$ after applying the difference operator $d_{t}$ to (B.1) and $v_{h}=s(\varepsilon) d_{t} U^{m}$ in (3.2). Then add the resulting equations. The assertion immediately follows from taking summation over $m$, using Lemma 3.1 and the following inequalities:

$$
\begin{aligned}
& \left(d_{t} f\left(\Phi^{m}\right), d_{t} \Phi^{m}\right)=\left(f^{\prime}(\xi),\left|d_{t} \Phi^{m}\right|^{2}\right) \geq-\left\|d_{t} \Phi^{m}\right\|_{L^{2}}^{2}, \\
& \left|s(\varepsilon) k\left(d_{t}^{2} U^{m}, d_{t} \Phi^{m}\right)\right| \leq \frac{\varepsilon \alpha(\varepsilon) k}{8}\left\|d_{t}^{2} U^{m}\right\|_{L^{2}}^{2}+\frac{8 s(\varepsilon)^{2} k}{\varepsilon \alpha(\varepsilon)}\left\|d_{t} \Phi^{m}\right\|_{L^{2}}^{2} .
\end{aligned}
$$

In order to establish error bounds that depend on low order polynomials of $\frac{1}{\varepsilon}$, we need a discrete version of the spectral estimate of Lemma 2.3. To that end, we define

$$
C_{1}=\max _{|\xi| \leq 2 C_{0}}\left|f^{\prime \prime}(\xi)\right|,
$$

and $C_{2}$ is the smallest positive $\varepsilon$-independent constant such that

$$
\begin{aligned}
\underset{J}{\operatorname{ess} \sup _{J}\left\|\varphi-P_{h} \varphi\right\|_{L^{\infty}}} & \leq C_{2} h^{\frac{4-N}{2}}|\ln h|^{\frac{3-N}{2}} \operatorname{ess} \sup _{J}\|\varphi\|_{H^{2}} \\
& \leq C_{2} h^{\frac{4-N}{2}}|\ln h|^{\frac{3-N}{2}} \mathcal{B}_{2}^{\frac{1}{2}} .
\end{aligned}
$$

Lemma 3.3. Suppose that the assumptions of Lemmas 2.12 .3 hold and that $C_{0}$ and $\varepsilon_{0}$ are the same as there. Then for $\varepsilon \in\left(0, \varepsilon_{0}\right]$ it holds that

$$
\begin{aligned}
\lambda_{P F}^{h} \equiv \inf _{\substack{\psi \in H^{1}(\Omega) \\
w \in H^{2}(\Omega)}}\left\{\frac{\varepsilon\|\nabla \psi\|_{L^{2}}^{2}}{\varepsilon \alpha(\varepsilon)\|\psi\|_{L^{2}}^{2}+s(\varepsilon)\|\nabla w\|_{L^{2}}^{2}}\right. \\
\left.+\frac{\frac{1}{\varepsilon}\left(f^{\prime}\left(P_{h} \varphi\right) \psi, \psi\right)+\frac{s(\varepsilon)}{c(\varepsilon)}\|\Delta w-\psi\|_{L^{2}}^{2}}{\varepsilon \alpha(\varepsilon)\|\psi\|_{L^{2}}^{2}+s(\varepsilon)\|\nabla w\|_{L^{2}}^{2}}\right\}
\end{aligned}
$$

provided that $h$ satisfies

$$
h^{\frac{4-N}{2}}|\ln h|^{\frac{3-N}{2}} \leq\left(C_{1} C_{2} \mathcal{B}_{2}^{\frac{1}{2}}\right)^{-1} C_{0} \alpha(\varepsilon) \varepsilon^{2} .
$$

In the above, $\varphi$ denotes the first component of the solution vector $(\varphi, u)$ to the phase field model (1.1)-1.4).

Proof. From the definition of $C_{1}$ and $C_{2}$, we immediately have

$$
\begin{aligned}
& \operatorname{ess} \sup _{J}\left\|P_{h} \varphi\right\|_{L^{\infty}} \leq \operatorname{ess} \sup _{J}\left\{\|\varphi\|_{L^{\infty}}+\left\|\varphi-P_{h} \varphi\right\|_{L^{\infty}}\right\} \\
& \leq \frac{4}{3} \operatorname{ess} \sup _{J}\|\varphi\|_{L^{\infty}} \leq 2 C_{0},
\end{aligned}
$$

provided that $h$ satisfies (3.15).

By the Mean Value Theorem,

$$
\begin{aligned}
\operatorname{ess} \sup _{J}\left\|f^{\prime}\left(P_{h} \varphi\right)-f^{\prime}(\varphi)\right\|_{L^{\infty}} & \leq \sup _{|\xi| \leq 2 C_{0}}\left|f^{\prime \prime}(\xi)\right| \operatorname{ess} \sup _{J}\left\|\varphi-P_{h} \varphi\right\|_{L^{\infty}} \\
& \leq C_{1} C_{2} h^{\frac{4-N}{2}}|\ln h|^{\frac{3-N}{2}} \mathcal{B}_{2}^{\frac{1}{2}} \\
& \leq C_{0} \alpha(\varepsilon) \varepsilon^{2} .
\end{aligned}
$$


Using the inequality $a \geq b-|a-b|$ and (3.16) , we get

$$
f^{\prime}\left(P_{h} \varphi\right) \geq f^{\prime}(\varphi)-\left|f^{\prime}\left(P_{h} \varphi\right)-f^{\prime}(\varphi)\right| \geq f^{\prime}(\varphi)-C_{0} \alpha(\varepsilon) \varepsilon^{2} .
$$

Substituting (3.17) into the definition of $\lambda_{P F}^{h}$, we get

$$
\begin{aligned}
\lambda_{P F}^{h} & \geq \inf _{\substack{\psi \in H^{1}(\Omega) \\
w \in H^{2}(\Omega)}}\left\{\frac{\varepsilon\|\nabla \psi\|_{L^{2}}^{2}+\frac{1}{\varepsilon}\left(f^{\prime}(\varphi) \psi, \psi\right)+\frac{s(\varepsilon)}{c(\varepsilon)}\|\Delta w-\psi\|_{L^{2}}^{2}}{\varepsilon \alpha(\varepsilon)\|\psi\|_{L^{2}}^{2}+s(\varepsilon)\|\nabla w\|_{L^{2}}^{2}}\right. \\
& \left.-\frac{C_{0} \alpha(\varepsilon) \varepsilon\|\psi\|_{L^{2}}^{2}}{\varepsilon \alpha(\varepsilon)\|\psi\|_{L^{2}}^{2}+s(\varepsilon)\|\nabla w\|_{L^{2}}^{2}}\right\} \\
& -2 C_{0} .
\end{aligned}
$$

The proof is completed.

We now state our first set of error estimates for the fully discrete scheme (3.1) (3.2), which will be shown by using some nonstandard test functions. To reduce some technicalities, we will only present the derivation of error bounds for the case $\varphi_{t t} \in L^{2}\left(J ; L^{2}\right)$ and $u_{t t} \in L^{2}\left(J ; L^{2}\right)$, and we will leave the derivation for the case $\varphi_{t t} \in L^{2}\left(J ; H^{-1}\right)$ and $u_{t t} \in L^{2}\left(J ; H^{-1}\right)$ to interested readers (cf. [26]).

Theorem 3.1. Let $\left\{\left(\Phi^{m}, U^{m}\right)\right\}_{m=0}^{M}$ solve (3.1)-(3.2) on a quasi-uniform time mesh $J_{k}:=\left\{t_{m}\right\}_{m=0}^{M}$ of size $O(k)$ and a quasi-uniform space mesh $\mathcal{T}_{h}$ of size $O(h)$. Suppose (GA) and (2.6) hold, and suppose the free boundary problem (1.7)-(1.12) has a unique classical solution. Then under the following mesh and starting value constraints

(1) $k \leq \min \left\{1, \alpha(\varepsilon)^{2}\right\} \times$

(4) $\left\|\Phi^{0}-\varphi_{0}^{\varepsilon}\right\|_{L^{2}} \leq C h^{2}\left\|\varphi_{0}^{\varepsilon}\right\|_{H^{2}}$,

(5) $\quad\left\|U^{0}-u_{0}^{\varepsilon}\right\|_{L^{2}} \leq C h^{2}\left\|u_{0}^{\varepsilon}\right\|_{H^{2}}$,

for $N=2,3$, the solution of (3.1)-(3.2) satisfies the error estimates

$$
\max _{0 \leq m \leq M} \sqrt{\varepsilon \alpha(\varepsilon)}\left\|\varphi\left(t_{m}\right)-\Phi^{m}\right\|_{L^{2}} \leq C\left[\rho_{1}(\varepsilon)^{\frac{1}{2}} h^{2}+\eta(\varepsilon)^{\frac{1}{2}} h^{2}+\zeta(\varepsilon)^{\frac{1}{2}} k\right]
$$

$$
\left\{c(\varepsilon) s(\varepsilon) k \sum_{m=0}^{M}\left\|u\left(t_{m}\right)-U^{m}\right\|_{L^{2}}^{2}\right\}^{\frac{1}{2}} \leq C\left[\rho_{2}(\varepsilon)^{\frac{1}{2}} h^{2}+\eta(\varepsilon)^{\frac{1}{2}} h^{2}+\zeta(\varepsilon)^{\frac{1}{2}} k\right],
$$

$$
\max _{0 \leq m \leq M}\left\|k \sum_{j=0}^{m} \nabla\left(u\left(t_{m}\right)-U^{m}\right)\right\|_{L^{2}} \leq C\left[\rho_{3}(\varepsilon)^{\frac{1}{2}} h+\eta(\varepsilon)^{\frac{1}{2}} h^{2}+\zeta(\varepsilon)^{\frac{1}{2}} k\right],
$$


(iv)

$$
\begin{gathered}
\text { (iv) } \quad\left\{\varepsilon^{3} \alpha(\varepsilon) k \sum_{m=1}^{M}\left\|\nabla\left(\varphi\left(t_{m}\right)-\Phi^{m}\right)\right\|_{L^{2}}^{2}\right\}^{\frac{1}{2}} \leq C\left[\rho_{4}(\varepsilon)^{\frac{1}{2}} h+\eta(\varepsilon)^{\frac{1}{2}} h^{2}+\zeta(\varepsilon)^{\frac{1}{2}} k\right], \\
\text { (v) } \sqrt{\varepsilon \alpha(\varepsilon)} \max _{0 \leq m \leq M}\left\|\varphi\left(t_{m}\right)-\Phi^{m}\right\|_{L^{\infty}} \\
\leq C\left\{\rho_{1}(\varepsilon)^{\frac{1}{2}} h^{\frac{4-N}{2}}|\ln h|^{\frac{3-N}{2}}+\left[\eta(\varepsilon)^{\frac{1}{2}} h^{2}+\zeta(\varepsilon)^{\frac{1}{2}} k\right] h^{-\frac{N}{2}}\right\},
\end{gathered}
$$

where $\rho_{i}(\varepsilon), \zeta(\varepsilon)$ and $\eta(\varepsilon)$ are defined by

$$
\begin{aligned}
& \rho_{1}(\varepsilon)=\varepsilon \alpha(\varepsilon) \mathcal{B}_{2}, \\
& \rho_{2}(\varepsilon)=c(\varepsilon) s(\varepsilon)\left\{\mathcal{J}_{\varepsilon}\left(\varphi_{0}^{\varepsilon}, u_{0}^{\varepsilon}\right)+[1+c(\varepsilon)] \varepsilon^{-2 \sigma}\right\}, \\
& \rho_{3}(\varepsilon)=\mathcal{J}_{\varepsilon}\left(\varphi_{0}^{\varepsilon}, u_{0}^{\varepsilon}\right)+[1+c(\varepsilon)] \varepsilon^{-2 \sigma}, \\
& \rho_{4}(\varepsilon)=\varepsilon^{3} \alpha(\varepsilon)\left[\frac{\alpha(\varepsilon)}{\varepsilon^{2}}+\varepsilon s(\varepsilon) c(\varepsilon)\right] \mathcal{J}_{\varepsilon}\left(\varphi_{0}^{\varepsilon}, u_{0}^{\varepsilon}\right), \\
& \text { (3.18) } \eta(\varepsilon)=\left[\frac{2 \varepsilon^{2} s(\varepsilon)^{2}+c(\varepsilon)^{2}}{\varepsilon^{3} c(\varepsilon)^{2} \alpha(\varepsilon)}+\frac{s(\varepsilon)}{\varepsilon^{2} c(\varepsilon) \alpha(\varepsilon)}\right]\left[\frac{\alpha(\varepsilon)}{\varepsilon^{2}}+\varepsilon s(\varepsilon) \alpha(\varepsilon)\right] \mathcal{J}_{\varepsilon}\left(\varphi_{0}^{\varepsilon}, u_{0}^{\varepsilon}\right) \\
& +\frac{s(\varepsilon)[2 \varepsilon s(\varepsilon)+c(\varepsilon) \alpha(\varepsilon)]}{\varepsilon^{2} \alpha(\varepsilon)}\left\{\varepsilon^{-2 \sigma_{4}}+\mathcal{J}_{\varepsilon}\left(\varphi_{0}^{\varepsilon}, u_{0}^{\varepsilon}\right)+[1+c(\varepsilon)] \varepsilon^{-2 \sigma_{3}}\right\} \\
& +\frac{s(\varepsilon)^{2}\left\{\mathcal{J}_{\varepsilon}\left(\varphi_{0}^{\varepsilon}, u_{0}^{\varepsilon}\right)+C[1+c(\varepsilon)] \varepsilon^{-2 \sigma_{3}}\right\}}{\varepsilon \alpha(\varepsilon)}+\frac{\alpha(\varepsilon)^{2} \mathcal{B}_{7}}{\varepsilon} \\
& +\frac{s(\varepsilon)[2 \varepsilon s(\varepsilon)+c(\varepsilon)] \varepsilon^{-2\left(\sigma_{2}+1\right)}}{\varepsilon^{2} c(\varepsilon)^{2} \alpha(\varepsilon)}+\frac{\mathcal{B}_{2}^{\frac{3}{2}}}{\varepsilon}\left\{1+\left[\mathcal{B}_{2}+\frac{\mathcal{B}_{1}}{\alpha(\varepsilon)}\right]^{\frac{N}{8}} \mathcal{B}_{2}^{-\frac{N}{8}}\right\} h^{\frac{4-N}{2}} ; \\
& (3.19) \zeta(\varepsilon)=\left[\varepsilon \alpha(\varepsilon)+\frac{s(\varepsilon)}{\varepsilon^{2} c(\varepsilon) \alpha(\varepsilon)}\right] \mathcal{B}_{7}+\frac{s(\varepsilon)^{2} k}{\varepsilon \alpha(\varepsilon)}\left\{\frac{\mathcal{J}_{\varepsilon}\left(\varphi_{0}^{\varepsilon}, u_{0}^{\varepsilon}\right)+[1+c(\varepsilon)] \varepsilon^{-2 \sigma_{3}}}{c(\varepsilon)^{2}}\right. \\
& \left.+\frac{\left[1+s(\varepsilon)^{2} \varepsilon^{3}\right] \mathcal{J}_{\varepsilon}\left(\varphi_{0}^{\varepsilon}, u_{0}^{\varepsilon}\right)+s(\varepsilon) \varepsilon^{-2 \sigma_{3}+1}}{c(\varepsilon) s(\varepsilon) \varepsilon}\right\}+\frac{s(\varepsilon)}{\varepsilon^{2} \alpha(\varepsilon)} \mathcal{B}_{8} ;
\end{aligned}
$$

Proof. The proof is divided into four steps. The first step estimates the consistency error of the scheme; the second and third steps deal with the error due to the nonlinear term $f(\varphi)$ and how to bound it in terms of some low order polynomial in $\frac{1}{\varepsilon}$, with the help of the stability estimates of Lemmas 3.1 and 3.2 and the spectral estimates of Lemma 3.3 the final step employs an inductive argument to handle the super-quadratic (cubic) term in $(f(a)-f(b), a-b)$.

Step 1. We decompose the global errors $E_{\varphi}^{m}:=\varphi\left(t_{m}\right)-\Phi^{m}$ and $E_{u}^{m}:=u\left(t_{m}\right)-$ $U^{m}$ into

$$
E_{\varphi}^{m}:=\Theta_{\varphi}^{m}+\Upsilon_{\varphi}^{m}, \quad E_{u}^{m}:=\Theta_{u}^{m}+\Upsilon_{u}^{m}
$$

where

$$
\begin{array}{rll}
\Theta_{\varphi}^{m}:=\varphi\left(t_{m}\right)-P_{h} \varphi\left(t_{m}\right), & \Upsilon_{\varphi}^{m}=P_{h} \varphi\left(t_{m}\right)-\Phi^{m} \\
\Theta_{u}^{m}:=u\left(t_{m}\right)-P_{h} u\left(t_{m}\right), & \Upsilon_{u}^{m}=P_{h} u\left(t_{m}\right)-U^{m}
\end{array}
$$


Then the error equations are given by

$$
\begin{gathered}
\varepsilon \alpha(\varepsilon)\left(d_{t} \Upsilon_{\varphi}^{m}, \eta_{h}\right)+\varepsilon\left(\nabla \Upsilon_{\varphi}^{m}, \nabla \eta_{h}\right)+\frac{1}{\varepsilon}\left(f\left(P_{h} \varphi\left(t_{m}\right)\right)-f\left(\Phi^{m}\right), \eta_{h}\right) \\
=-\varepsilon \alpha(\varepsilon)\left(d_{t} \Theta_{\varphi}^{m}, \eta_{h}\right)+s(\varepsilon)\left(\Theta_{u}^{m-1}+\Upsilon_{u}^{m}, \eta_{h}\right) \\
+k s(\varepsilon)\left(d_{t} u\left(t_{m}\right), \eta_{h}\right)-s(\varepsilon) k\left(d_{t} \Upsilon_{u}^{m}, \eta_{h}\right) \\
\quad-\frac{1}{\varepsilon}\left(f\left(\varphi\left(t_{m}\right)\right)-f\left(P_{h} \varphi\left(t_{m}\right)\right), \eta_{h}\right)+\varepsilon \alpha(\varepsilon)\left(\mathcal{R}_{\varphi}^{m}, \eta_{h}\right) . \\
c(\varepsilon)\left(d_{t} \Upsilon_{u}^{m}, v_{h}\right)+\left(\nabla \Upsilon_{u}^{m}, \nabla v_{h}\right) \\
=-\left(d_{t} \Upsilon_{\varphi}^{m}+d_{t} \Theta_{\varphi}^{m}, v_{h}\right)-c(\varepsilon)\left(d_{t} \Theta_{u}^{m}, v_{h}\right)+\left(c(\varepsilon) \mathcal{R}_{u}^{m}+\mathcal{R}_{\varphi}^{m}, v_{h}\right),
\end{gathered}
$$

for all $\left(\eta_{h}, v_{h}\right) \in\left[V_{h}\right]^{2}$, where

$$
\mathcal{R}_{u}^{m}=-\frac{1}{k} \int_{t_{m}}^{t_{m+1}}\left(s-t_{m}\right) u_{t t}(s) \mathrm{d} s, \quad \mathcal{R}_{\varphi}^{m}=-\frac{1}{k} \int_{t_{m}}^{t_{m+1}}\left(s-t_{m}\right) \varphi_{t t}(s) \mathrm{d} s .
$$

Using Schwartz's inequality, we have

$$
k \sum_{m=0}^{\ell}\left\|\mathcal{R}_{\varphi}^{m}\right\|_{L^{2}}^{2} \leq C k^{2} \mathcal{B}_{7}, \quad k \sum_{m=0}^{\ell}\left\|\mathcal{R}_{u}^{m}\right\|_{L^{2}}^{2} \leq \frac{C k^{2}}{c(\varepsilon)} \mathcal{B}_{8} .
$$

Step 2. In the sequel, it turns out that the most crucial term to handle is $s(\varepsilon)\left(\Upsilon_{u}^{m}, \eta_{h}\right)$ in (3.21). Dealing with it requires a preparatory step: first, replacing the super-index $m$ in (3.22) by $j$ and then summing the resulting equation over $1 \leq j \leq m$ yields

$$
c(\varepsilon)\left(\Upsilon_{u}^{m}, v_{h}\right)+\left(\nabla G_{u}^{m}, \nabla v_{h}\right)=-\left(\Theta_{\varphi}^{m}+\Upsilon_{\varphi}^{m}, v_{h}\right)-c(\varepsilon)\left(\Theta_{u}^{m}, v_{h}\right)+c(\varepsilon)\left(E_{u}^{0}, v_{h}\right)
$$

$$
+\left(E_{\varphi}^{0}, v_{h}\right)+\left(k \sum_{j=1}^{m}\left\{c(\varepsilon) \mathcal{R}_{u}^{j}+\mathcal{R}_{\varphi}^{j}\right\}, v_{h}\right)
$$

where

$$
G_{u}^{0}=0 \quad \text { and } \quad G_{u}^{m}=k \sum_{j=1}^{m} \Upsilon_{u}^{j} .
$$

Set $v_{h}=\Upsilon_{\varphi}^{m}$ in (3.25) and substitute it into the right-hand side of (3.21) with $\eta_{h}=\Upsilon_{\varphi}^{m}$. We get

$$
\begin{gathered}
\frac{\varepsilon \alpha(\varepsilon)}{2}\left[d_{t}\left\|\Upsilon_{\varphi}^{m}\right\|_{L^{2}}^{2}+k\left\|d_{t} \Upsilon_{\varphi}^{m}\right\|_{L^{2}}^{2}\right]+\varepsilon\left\|\nabla \Upsilon_{\varphi}^{m}\right\|_{L^{2}}^{2} \\
+\frac{1}{\varepsilon}\left(f\left(P_{h} \varphi\left(t_{m}\right)\right)-f\left(\Phi^{m}\right), \Upsilon_{\varphi}^{m}\right)+\frac{s(\varepsilon)}{c(\varepsilon)}\left[\left\|\Upsilon_{\varphi}^{m}\right\|_{L^{2}}^{2}+\left(\nabla G_{u}^{m}, \nabla \Upsilon_{\varphi}^{m}\right)\right] \\
=-\varepsilon \alpha(\varepsilon)\left(d_{t} \Theta_{\varphi}^{m}, \Upsilon_{\varphi}^{m}\right)+s(\varepsilon)\left(\Theta_{u}^{m-1}, \Upsilon_{\varphi}^{m}\right)-\frac{s(\varepsilon)}{c(\varepsilon)}\left(\Theta_{\varphi}^{m}, \Upsilon_{\varphi}^{m}\right) \\
-s(\varepsilon)\left(\Theta_{u}^{m}, \Upsilon_{\varphi}^{m}\right)+s(\varepsilon)\left(E_{u}^{0}, \Upsilon_{\varphi}^{m}\right)+\frac{s(\varepsilon)}{c(\varepsilon)}\left(E_{\varphi}^{0}, \Upsilon_{\varphi}^{m}\right) \\
+\varepsilon \alpha(\varepsilon)\left(\mathcal{R}_{\varphi}^{m}, \Upsilon_{\varphi}^{m}\right)+\frac{s(\varepsilon)}{c(\varepsilon)}\left(k \sum_{j=0}^{m}\left[c(\varepsilon) \mathcal{R}_{u}^{j}+\mathcal{R}_{\varphi}^{j}\right], \Upsilon_{\varphi}^{m}\right) \\
-\frac{1}{\varepsilon}\left(f\left(\varphi\left(t_{m}\right)\right)-f\left(P_{h} \varphi\left(t_{m}\right)\right), \Upsilon_{\varphi}^{m}\right) \\
+k s(\varepsilon)\left(d_{t} u\left(t_{m}\right), \Upsilon_{\varphi}^{m}\right)-k s(\varepsilon)\left(d_{t} \Upsilon_{u}^{m}, \Upsilon_{\varphi}^{m}\right)
\end{gathered}
$$


In order to control the last term on the left-hand side of (3.26), we choose $v_{h}=-\frac{s(\varepsilon)}{c(\varepsilon)} \Delta_{h} G_{u}^{m}$ in (3.25) and obtain

$$
\begin{aligned}
& s(\varepsilon)\left(\Upsilon_{u}^{m},-\Delta_{h} G_{u}^{m}\right)+\frac{s(\varepsilon)}{c(\varepsilon)}\left[\left\|\Delta_{h} G_{u}^{m}\right\|_{L^{2}}^{2}-\left(\Upsilon_{\varphi}^{m}, \Delta_{h} G_{u}^{m}\right)\right] \\
& =\frac{s(\varepsilon)}{c(\varepsilon)}\left(\Theta_{\varphi}^{m}, \Delta_{h} G_{u}^{m}\right)+s(\varepsilon)\left[\left(\Theta_{u}^{m}, \Delta_{h} G_{u}^{m}\right)-\left(E_{u}^{0}, \Delta_{h} G_{u}^{m}\right)\right] \\
& \quad-\frac{s(\varepsilon)}{c(\varepsilon)}\left(E_{\varphi}^{0}, \Delta_{h} G_{u}^{m}\right)+\frac{s(\varepsilon)}{c(\varepsilon)}\left(k \sum_{j=1}^{m}\left[c(\varepsilon) \mathcal{R}_{u}^{j}+\mathcal{R}_{\varphi}^{j}\right], \Delta_{h} G_{u}^{m}\right) .
\end{aligned}
$$

Notice that $\Upsilon_{u}^{m}=d_{t} G_{u}^{m}$; hence the first term can be written as

$$
s(\varepsilon)\left(\Upsilon_{u}^{m},-\Delta_{h} G_{u}^{m}\right)=\frac{s(\varepsilon)}{2}\left[d_{t}\left\|\nabla G_{u}^{m}\right\|_{L^{2}}^{2}+k\left\|\nabla d_{t} G_{u}^{m}\right\|_{L^{2}}^{2}\right]
$$

Next, observe that

$$
\begin{aligned}
\left|\frac{1}{\varepsilon}\left(f\left(\varphi\left(t_{m}\right)\right)-f\left(P_{h} \varphi\left(t_{m}\right)\right), \Upsilon_{\varphi}^{m}\right)\right| & =\left|\frac{1}{\varepsilon}\left(f^{\prime}(\xi) \Theta_{\varphi}^{m}, \Upsilon_{\varphi}^{m}\right)\right| \\
& \leq \frac{\varepsilon \alpha(\varepsilon)}{4}\left\|\Upsilon_{\varphi}^{m}\right\|_{L^{2}}^{2}+\frac{C}{\varepsilon^{3} \alpha(\varepsilon)}\left\|\Theta_{\varphi}^{m}\right\|_{L^{2}}^{2},
\end{aligned}
$$

where we have used the fact that $\varphi$ and $P_{h} \varphi$ are bounded (cf. Lemma 2.1 and the first line of the proof of Lemma 3.3). Moreover, using the identity

$$
f(a)-f(b)=(a-b)\left[f^{\prime}(a)+(a-b)^{2}-3(a-b) a\right] \quad \forall a, b \in \mathbf{R},
$$

we get

$$
\begin{aligned}
\left(f\left(P_{h} \varphi\left(t_{m}\right)\right)-\right. & \left.f\left(\Phi^{m}\right), \Upsilon_{\varphi}^{m}\right) \\
& \geq\left(f^{\prime}\left(P_{h} \varphi\left(t_{m}\right)\right),\left(\Upsilon_{\varphi}^{m}\right)^{2}\right)+\left\|\Upsilon_{\varphi}^{m}\right\|_{L^{4}}^{4}-C\left\|\Upsilon_{\varphi}^{m}\right\|_{L^{3}}^{3} .
\end{aligned}
$$

Now, adding (3.26) and (3.27) and combining with (3.28) and (3.29) results in

$$
\begin{aligned}
\frac{\varepsilon \alpha(\varepsilon)}{2} d_{t}\left\|\Upsilon_{\varphi}^{m}\right\|_{L^{2}}^{2}+\frac{s(\varepsilon)}{2} d_{t}\left\|\nabla G_{u}^{m}\right\|_{L^{2}}^{2}+\frac{\varepsilon \alpha(\varepsilon) k}{2}\left\|d_{t} \Upsilon_{\varphi}^{m}\right\|_{L^{2}}^{2} & +\frac{s(\varepsilon) k}{2}\left\|\nabla \Upsilon_{u}^{m}\right\|_{L^{2}}^{2}+\frac{1}{\varepsilon}\left\|\Upsilon_{\varphi}^{m}\right\|_{L^{4}}^{4}+\varepsilon\left\|\nabla \Upsilon_{\varphi}^{m}\right\|_{L^{2}}^{2} \\
& +\frac{1}{\varepsilon}\left(f^{\prime}\left(P_{h} \varphi\left(t_{m}\right)\right),\left(\Upsilon_{\varphi}^{m}\right)^{2}\right)+\frac{s(\varepsilon)}{c(\varepsilon)}\left\|\Upsilon_{\varphi}^{m}-\Delta_{h} G_{u}^{m}\right\|_{L^{2}}^{2} \\
\leq & \varepsilon \alpha(\varepsilon)\left[\left(d_{t} \Theta_{\varphi}^{m}, \Upsilon_{\varphi}^{m}\right)+\left(\mathcal{R}_{\varphi}^{m}, \Upsilon_{\varphi}^{m}\right)\right]+s(\varepsilon)\left(\Theta_{u}^{m-1}, \Upsilon_{\varphi}^{m}\right) \\
+ & s(\varepsilon)\left[\left(E_{u}^{0}, \Upsilon_{\varphi}^{m}\right)-\left(\Theta_{u}^{m}, \Upsilon_{\varphi}^{m}\right)\right]+\frac{s(\varepsilon)}{c(\varepsilon)}\left[\left(E_{\varphi}^{0}, \Upsilon_{\varphi}^{m}\right)-\left(\Theta_{\varphi}^{m}, \Upsilon_{\varphi}^{m}\right)\right] \\
+ & \frac{s(\varepsilon)}{c(\varepsilon)}\left[\left(k \sum_{j=0}^{m}\left[c(\varepsilon) \mathcal{R}_{u}^{j}+\mathcal{R}_{\varphi}^{j}\right], \Upsilon_{\varphi}^{m}-\Delta_{h} G_{u}^{m}\right)+\left(\Theta_{\varphi}^{m}, \Delta_{h} G_{u}^{m} \pm \Upsilon_{\varphi}^{m}\right)\right] \\
+ & s(\varepsilon)\left[\left(\Theta_{u}^{m}, \Delta_{h} G_{u}^{m} \pm \Upsilon_{\varphi}^{m}\right)-\left(E_{u}^{0}, \Delta_{h} G_{u}^{m} \pm \Upsilon_{\varphi}^{m}\right)\right] \\
- & \frac{s(\varepsilon)}{c(\varepsilon)}\left(E_{\varphi}^{0}, \Delta_{h} G_{u}^{m} \pm \Upsilon_{\varphi}^{m}\right)+k s(\varepsilon)\left(d_{t} u\left(t_{m}\right), \Upsilon_{\varphi}^{m}\right) \\
- & s(\varepsilon) k\left(d_{t} \Upsilon_{u}^{m}, \Upsilon_{\varphi}^{m}\right)+\frac{\varepsilon \alpha(\varepsilon)}{4}\left\|\Upsilon_{\varphi}^{m}\right\|_{L^{2}}^{2}+\frac{C}{\varepsilon^{3} \alpha(\varepsilon)}\left\|\Theta_{\varphi}^{m}\right\|_{L^{2}}^{2}+\frac{C}{\varepsilon}\left\|\Upsilon_{\varphi}^{m}\right\|_{L^{3}}^{3}
\end{aligned}
$$


Using Young's inequality, the right-hand side of (3.30), denoted by $S_{1}^{m}$, can be bounded by (after taking summation over $m$ )

$$
\begin{gathered}
\left|k \sum_{m=1}^{\ell} S_{1}^{m}\right| \leq C\left[\eta_{1}(\varepsilon) h^{4}+\zeta(\varepsilon) k^{2}\right]+k \sum_{m=1}^{\ell}\left\{\frac{\varepsilon^{2} \alpha(\varepsilon) s(\varepsilon)}{2 c(\varepsilon)}\left\|\Upsilon_{\varphi}^{m}-\Delta_{h} G_{u}^{m}\right\|_{L^{2}}^{2}\right. \\
\left.+\frac{\varepsilon \alpha(\varepsilon)}{2}\left\|\Upsilon_{\varphi}^{m}\right\|_{L^{2}}^{2}+\frac{C}{\varepsilon}\left\|\Upsilon_{\varphi}^{m}\right\|_{L^{3}}^{3}\right\}
\end{gathered}
$$

where $\zeta(\varepsilon)$ is defined by (3.19) and $\eta_{1}(\varepsilon)$ has the form

$$
\begin{aligned}
\eta_{1}(\varepsilon)= & {\left[\frac{2 \varepsilon^{2} s(\varepsilon)^{2}+c(\varepsilon)^{2}}{\varepsilon^{3} c(\varepsilon)^{2} \alpha(\varepsilon)}+\frac{s(\varepsilon)}{\varepsilon^{2} c(\varepsilon) \alpha(\varepsilon)}\right]\left[\frac{\alpha(\varepsilon)}{\varepsilon^{2}}+\varepsilon s(\varepsilon) \alpha(\varepsilon)\right] \mathcal{J}_{\varepsilon}\left(\varphi_{0}^{\varepsilon}, u_{0}^{\varepsilon}\right) } \\
& +\frac{s(\varepsilon)[2 \varepsilon s(\varepsilon)+c(\varepsilon) \alpha(\varepsilon)]}{\varepsilon^{2} \alpha(\varepsilon)}\left\{\varepsilon^{-2 \sigma_{4}}+\mathcal{J}_{\varepsilon}\left(\varphi_{0}^{\varepsilon}, u_{0}^{\varepsilon}\right)+[1+c(\varepsilon)] \varepsilon^{-2 \sigma_{3}}\right\} \\
& +\frac{s(\varepsilon)^{2}\left\{\mathcal{J}_{\varepsilon}\left(\varphi_{0}^{\varepsilon}, u_{0}^{\varepsilon}\right)+C[1+c(\varepsilon)] \varepsilon^{-2 \sigma_{3}}\right\}}{\varepsilon \alpha(\varepsilon)}+\frac{\alpha(\varepsilon)^{2} \widetilde{\mathcal{B}}_{7}}{\varepsilon} \\
& +\frac{s(\varepsilon)[2 \varepsilon s(\varepsilon)+c(\varepsilon)] \varepsilon^{-2\left(\sigma_{2}+1\right)}}{\varepsilon^{2} c(\varepsilon)^{2} \alpha(\varepsilon)}
\end{aligned}
$$

Substituting (3.31) into (3.30) after taking summation over $m$ leads to

$$
\begin{aligned}
\frac{\varepsilon \alpha(\varepsilon)}{2} \| & \Upsilon_{\varphi}^{\ell}\left\|_{L^{2}}^{2}+\frac{s(\varepsilon)}{2}\right\| \nabla G_{u}^{\ell} \|_{L^{2}}^{2}+k \sum_{m=1}^{\ell}\left\{\frac{\varepsilon \alpha(\varepsilon) k}{2}\left\|d_{t} \Upsilon_{\varphi}^{m}\right\|_{L^{2}}^{2}\right. \\
& +\frac{s(\varepsilon) k}{2}\left\|\nabla \Upsilon_{u}^{m}\right\|_{L^{2}}^{2}+\frac{1}{\varepsilon}\left\|\Upsilon_{\varphi}^{m}\right\|_{L^{4}}^{4}+\varepsilon\left\|\nabla \Upsilon_{\varphi}^{m}\right\|_{L^{2}}^{2} \\
& \left.+\frac{1}{\varepsilon}\left(f^{\prime}\left(P_{h} \varphi\left(t_{m}\right)\right),\left(\Upsilon_{\varphi}^{m}\right)^{2}\right)+\frac{s(\varepsilon)}{c(\varepsilon)}\left[1-\frac{\varepsilon^{2} \alpha(\varepsilon)}{2}\right]\left\|\Upsilon_{\varphi}^{m}-\Delta_{h} G_{u}^{m}\right\|_{L^{2}}^{2}\right\} \\
\leq & C\left[\eta_{1}(\varepsilon) h^{4}+\zeta(\varepsilon) k^{2}\right]+k \sum_{m=0}^{\ell}\left\{\frac{\varepsilon \alpha(\varepsilon)}{2}\left\|\Upsilon_{\varphi}^{m}\right\|_{L^{2}}^{2}+\frac{C}{\varepsilon}\left\|\Upsilon_{\varphi}^{m}\right\|_{L^{3}}^{3}\right\} .
\end{aligned}
$$

Step 3. Two terms in (3.33) remain to be bounded, namely, the first term on the third line and the last term on the right-hand side. In the following, we will bound the first one from below using the discrete spectrum estimate (3.14), and we will bound the second from above using a spatial-temporal decomposition technique.

First, notice that $\Delta_{h}$, not $\Delta$, appears in (3.33); in order to use (3.14), we need a preparatory step. Let $\mathcal{F}_{\lambda}[\cdot]$ denote the convolution operator with a mollifier as defined in [1] -it is well known that for any $v \in H^{r}(\Omega)(r \geq 0), \mathcal{F}_{\lambda}[v] \in C_{0}^{\infty}\left(\mathbf{R}^{N}\right)$ converges to $v$ in the $H^{r}$ norm as $\lambda \searrow 0$; in particular, for any $\delta>0$ there exists a $\lambda(\delta)>0$ such that for $0<\lambda<\lambda(\delta)$

$$
\left\|v-\mathcal{F}_{\lambda}[v]\right\|_{H^{r}} \leq \delta\|v\|_{H^{r}} \quad \forall v \in H^{r}(r \geq 0) .
$$

Now using the identity

$$
\begin{aligned}
& \left\|\Upsilon_{\varphi}^{m}-\Delta_{h} G_{u}^{m}\right\|_{L^{2}}^{2} \\
& =\left\|\Upsilon_{\varphi}^{m}-\Delta \mathcal{F}_{\lambda}\left[G_{u}^{m}\right]\right\|_{L^{2}}^{2}+\left(\Delta \mathcal{F}_{\lambda}\left[G_{u}^{m}\right]-\Delta_{h} G_{u}^{m}, \Upsilon_{\varphi}^{m}-\Delta_{h} G_{u}^{m}\right) \\
& \quad+\left(\Delta \mathcal{F}_{\lambda}\left[G_{u}^{m}\right]-\Delta_{h} G_{u}^{m}, \Upsilon_{\varphi}^{m}-\Delta \mathcal{F}_{\lambda}\left[G_{u}^{m}\right]\right),
\end{aligned}
$$


we conclude that there exists $\lambda(\varepsilon)>0$ such that for $\lambda<\lambda(\varepsilon)$

$$
\begin{aligned}
& \Upsilon_{\varphi}^{m}-\Delta_{h} G_{u}^{m}\left\|_{L^{2}}^{2} \geq\right\| \Upsilon_{\varphi}^{m}-\Delta \mathcal{F}_{\lambda}\left[G_{u}^{m}\right] \|_{L^{2}}^{2} \\
& -\frac{\varepsilon^{2} \alpha(\varepsilon) s(\varepsilon)}{4 c(\varepsilon)}\left\|\Upsilon_{\varphi}^{m}-\Delta_{h} G_{u}^{m}\right\|_{L^{2}}^{2}-\frac{s(\varepsilon)}{2}\left\|\nabla G_{u}^{m}\right\|_{L^{2}}^{2}-\frac{\varepsilon^{3} \alpha(\varepsilon)}{4}\left\|\nabla \Upsilon_{\varphi}^{m}\right\|_{L^{2}}^{2}
\end{aligned}
$$

To see (3.35), we deal with the last two terms in (3.34) separately. Taking into account that $\Upsilon_{\varphi}^{m}-\Delta_{h} G_{u}^{m} \in V_{h}$, we first conclude that for an appropriately chosen $\tilde{\delta}_{1}=\tilde{\delta}_{1}(\delta)>0$,

$$
\begin{gathered}
\left(\Delta \mathcal{F}_{\lambda}\left[G_{u}^{m}\right]-\Delta_{h} G_{u}^{m}, \Upsilon_{\varphi}^{m}-\Delta_{h} G_{u}^{m}\right)=\left(\nabla\left[\mathcal{F}_{\lambda}\left[G_{u}^{m}\right]-G_{u}^{m}\right], \nabla\left[\Upsilon_{\varphi}^{m}-\Delta_{h} G_{u}^{m}\right]\right) \\
\leq \tilde{\delta}_{1}\left\|\nabla G_{u}^{m}\right\|_{L^{2}}\left\|\nabla\left[\Upsilon_{\varphi}^{m}-\Delta_{h} G_{u}^{m}\right]\right\|_{L^{2}} .
\end{gathered}
$$

For the second term, we proceed independently for every $K \in \mathcal{T}_{h}$, and we benefit from the fact that $\left.\left(\Delta G_{u}^{m}\right)\right|_{K}=0$. In the following, we choose $\tilde{\delta}_{2}=\tilde{\delta}_{2}(\delta)>0$ appropriately such that

$$
\begin{aligned}
\sum_{K \in \mathcal{T}_{h}}\left(\Delta \mathcal{F}_{\lambda}\left[G_{u}^{m}\right]-\Delta_{h} G_{u}^{m}, \Upsilon_{\varphi}^{m}-\Delta \mathcal{F}_{\lambda}\left[G_{u}^{m}\right]\right)_{K} \\
=\sum_{K \in \mathcal{T}_{h}}\left[\left(\Delta \mathcal{F}_{\lambda}\left[G_{u}^{m}\right]-\Delta_{h} G_{u}^{m}, \Upsilon_{\varphi}^{m}\right)_{K}\right. \\
\left.\quad+\left(\Delta \mathcal{F}_{\lambda}\left[G_{u}^{m}\right]-\Delta_{h} G_{u}^{m}, \Delta\left[G_{u}^{m}-\mathcal{F}_{\lambda}\left[G_{u}^{m}\right]\right]\right)_{K}\right] \\
\leq\left(\nabla\left[G_{u}^{m}-\mathcal{F}_{\lambda}\left[G_{u}^{m}\right]\right], \nabla \Upsilon_{\varphi}^{m}\right) \\
\quad+\tilde{\delta}_{2} \sum_{K \in \mathcal{T}_{h}}\left\|\Delta \mathcal{F}_{\lambda}\left[G_{u}^{m}\right]-\Delta_{h} G_{u}^{m}\right\|_{L^{2}(K)}\left\|G_{u}^{m}\right\|_{H^{2}(K)} \\
\leq 2 \delta\left\|\nabla G_{u}^{m}\right\|_{L^{2}}\left\|\nabla \Upsilon_{\varphi}^{m}\right\|_{L^{2}} .
\end{aligned}
$$

Above, we used the following fact, which is valid for all $\varphi_{h} \in V_{h}$,

$$
\begin{aligned}
\sum_{K \in \mathcal{T}_{h}}\left\|\Delta_{h} \varphi_{h}\right\|_{L^{2}(K)}^{2} & =-\sum_{K \in \mathcal{T}_{h}}\left(\nabla \Delta_{h} \varphi_{h}, \nabla \varphi_{h}\right)_{K} \\
& \leq C \sum_{K \in \mathcal{T}_{h}} h_{K}^{-1}\left\|\Delta_{h} \varphi_{h}\right\|_{L^{2}(K)}\left\|\nabla \varphi_{h}\right\|_{L^{2}(K)} ;
\end{aligned}
$$

hence, $\left\|\Delta_{h} \varphi_{h}\right\|_{L^{2}} \leq \min _{K \in \mathcal{T}_{h}} h_{K}^{-1}\left\|\nabla \varphi_{h}\right\|_{L^{2}}$. In addition, we also utilized the following estimate

$$
\left\|\Delta \mathcal{F}_{\lambda}\left[G_{u}^{m}\right]\right\|_{L^{2}(K)} \leq\left(1+\tilde{\delta}_{2}\right)\left\|G_{u}^{m}\right\|_{H^{2}(K)} \leq \delta\left\|G_{u}^{m}\right\|_{H^{1}(K)},
$$

thanks to an inverse inequality. These arguments establish (3.35).

Next, it follows from (3.14) that there exists $\lambda_{0}>0$ such that for $\lambda<\lambda_{0}$,

$$
\begin{gathered}
{\left[1-\varepsilon^{2} \alpha(\varepsilon)\right]\left\{\varepsilon\left\|\Upsilon_{\varphi}^{m}\right\|_{L^{2}}^{2}+\frac{1}{\varepsilon}\left(f\left(P_{h} \varphi\left(t_{m}\right)\right),\left(\Upsilon_{\varphi}^{m}\right)^{2}\right)\right.} \\
\left.+\frac{s(\varepsilon)}{c(\varepsilon)}\left\|\Upsilon_{\varphi}^{m}-\Delta \mathcal{F}_{\lambda}\left[G_{u}^{m}\right]\right\|_{L^{2}}^{2}\right\} \\
\geq-2 C_{0}\left\{\varepsilon \alpha(\varepsilon)\left\|\Upsilon_{\varphi}^{m}\right\|_{L^{2}}^{2}+s(\varepsilon)\left\|\nabla \mathcal{F}_{\lambda}\left[G_{u}^{m}\right]\right\|_{L^{2}}^{2}\right\} \\
\geq-4 C_{0}\left\{\varepsilon \alpha(\varepsilon)\left\|\Upsilon_{\varphi}^{m}\right\|_{L^{2}}^{2}+s(\varepsilon)\left\|\nabla G_{u}^{m}\right\|_{L^{2}}^{2}\right\} .
\end{gathered}
$$


Substituting (3.35) and (3.36) into (3.33) gives

$$
\begin{gathered}
\frac{\varepsilon \alpha(\varepsilon)}{2}\left\|\Upsilon_{\varphi}^{\ell}\right\|_{L^{2}}^{2}+\frac{s(\varepsilon)}{2}\left\|\nabla G_{u}^{\ell}\right\|_{L^{2}}^{2}+k \sum_{m=1}^{\ell}\left\{\frac{\varepsilon \alpha(\varepsilon) k}{2}\left\|d_{t} \Upsilon_{\varphi}^{m}\right\|_{L^{2}}^{2}\right. \\
+\frac{s(\varepsilon) k}{2}\left\|\nabla \Upsilon_{u}^{m}\right\|_{L^{2}}^{2}+\frac{1}{\varepsilon}\left\|\Upsilon_{\varphi}^{m}\right\|_{L^{4}}^{4}+\frac{\varepsilon^{3} \alpha(\varepsilon)}{4}\left\|\nabla \Upsilon_{\varphi}^{m}\right\|_{L^{2}}^{2} \\
\left.+\frac{\varepsilon^{2} \alpha(\varepsilon) s(\varepsilon)}{4 c(\varepsilon)}\left\|\Upsilon_{\varphi}^{m}-\Delta_{h} G_{u}^{m}\right\|_{L^{2}}^{2}\right\} \\
\leq C\left[\eta_{1}(\varepsilon) h^{4}+\zeta(\varepsilon) k^{2}\right]+k \sum_{m=0}^{\ell}\left\{\frac{\varepsilon \alpha(\varepsilon)\left[C+8 C_{0}\right]}{2}\left\|\Upsilon_{\varphi}^{m}\right\|_{L^{2}}^{2}\right. \\
\left.+\frac{s(\varepsilon)\left[1+8 C_{0}\right]}{2}\left\|\nabla G_{u}^{m}\right\|_{L^{2}}^{2}+\frac{C}{\varepsilon}\left\|\Upsilon_{\varphi}^{m}\right\|_{L^{3}}^{3}\right\}
\end{gathered}
$$

where $\eta(\varepsilon)$ and $\zeta(\varepsilon)$ are defined by (3.19) and (3.18), respectively.

Finally, we apply a spatial-temporal decomposition argument to bound the last term of (3.37). For this purpose, we make a shift in the super-index and use the triangle inequality to get

$$
\left\|E_{\varphi}^{m}\right\|_{L^{3}}^{3} \leq \sum_{K \in \mathcal{T}_{h}}\left[k^{3}\left\|d_{t} E_{\varphi}^{m}\right\|_{L^{3}(K)}^{3}+\left\|E_{\varphi}^{m-1}\right\|_{L^{3}(K)}^{3}\right] .
$$

For each term of the second sum on the right-hand side of (3.38), we interpolate $L^{3}(K)$ between $L^{2}(K)$ and $H^{2}(K)$,

$$
\begin{aligned}
\left\|E_{\varphi}^{m-1}\right\|_{L^{3}(K)}^{3} & \leq C\left(\left\|\Delta E_{\varphi}^{m-1}\right\|_{L^{2}(K)}^{\frac{N}{4}}\left\|E_{\varphi}^{m-1}\right\|_{L^{2}(K)}^{\frac{12-N}{4}}+\left\|E_{\varphi}^{m-1}\right\|_{L^{2}(K)}^{3}\right) \\
& \leq C\left\|E_{\varphi}^{m-1}\right\|_{L^{2}(K)}^{\frac{12-N}{4}}\left(\left\|\Delta E_{\varphi}^{m-1}\right\|_{L^{2}(K)}^{\frac{N}{4}}+\left\|E_{\varphi}^{m-1}\right\|_{L^{2}(K)}^{\frac{N}{4}}\right) \\
& \leq C\left\|E_{\varphi}^{m-1}\right\|_{L^{2}(K)}^{\frac{12-N}{4}} \mathcal{B}_{2}^{\frac{N}{8}} .
\end{aligned}
$$

The last step follows from (v) of Lemma 2.2 and Lemma 3.1 .

Similarly, we can bound the first sum on the right-hand side of (3.38) as

$$
\begin{aligned}
k^{3}\left\|d_{t} E_{\varphi}^{m}\right\|_{L^{3}(K)}^{3} & \leq C k^{3}\left(\left\|\Delta d_{t} E_{\varphi}^{m}\right\|_{L^{2}(K)}^{\frac{N}{4}}\left\|d_{t} E_{\varphi}^{m}\right\|_{L^{2}(K)}^{\frac{12-N}{4}}+\left\|d_{t} E_{\varphi}^{m}\right\|_{L^{2}(K)}^{3}\right) \\
& \leq C k^{3}\left\|d_{t} E_{\varphi}^{m}\right\|_{L^{2}(K)}^{\frac{12-N}{4}}\left(\left\|\Delta d_{t} E_{\varphi}^{m}\right\|_{L^{2}(K)}^{\frac{N}{4}}+\left\|d_{t} E_{\varphi}^{m}\right\|_{L^{2}(K)}^{\frac{N}{4}}\right) \\
& \leq C\left[\mathcal{B}_{2}+\frac{\mathcal{B}_{1}}{\alpha(\varepsilon)}\right]^{\frac{N}{8}} k^{\frac{12-N}{4}}\left\|d_{t} E_{\varphi}^{m}\right\|_{L^{2}(K)}^{\frac{12-N}{4}} .
\end{aligned}
$$

Summing (3.39) and (3.40) over all $K \in \mathcal{T}_{h}$ and using the convexity of the function $g(s)=s^{r}$ for $r>1$ and $s \geq 0$ then leads to

$$
\left\|E_{\varphi}^{m}\right\|_{L^{3}}^{3} \leq C\left\|E_{\varphi}^{m-1}\right\|_{L^{2}}^{\frac{12-N}{4}} \mathcal{B}_{2}^{\frac{N}{8}}+C\left[\mathcal{B}_{2}+\frac{\mathcal{B}_{1}}{\alpha(\varepsilon)}\right]^{\frac{N}{8}} k^{\frac{12-N}{4}}\left\|d_{t} E_{\varphi}^{m}\right\|_{L^{2}}^{\frac{12-N}{4}}
$$


Because of (3.20) and Lemma 2.2 the above estimate leads to

$$
\begin{aligned}
\left\|\Upsilon_{\varphi}^{m}\right\|_{L^{3}}^{3} \leq & C\left\{\left\|\Upsilon_{\varphi}^{m-1}\right\|_{L^{2}}^{\frac{12-N}{4}} \mathcal{B}_{2}^{\frac{N}{8}}+\left[\mathcal{B}_{2}+\frac{\mathcal{B}_{1}}{\alpha(\varepsilon)}\right]^{\frac{N}{8}} k^{\frac{12-N}{4}}\left\|d_{t} \Upsilon_{\varphi}^{m}\right\|_{L^{2}}^{\frac{12-N}{4}}\right. \\
+ & \left\|\Theta_{\varphi}^{m-1}\right\|_{L^{2}}^{\frac{12-N}{4}} \mathcal{B}_{2}^{\frac{N}{8}}+\left[\mathcal{B}_{2}+\frac{\mathcal{B}_{1}}{\alpha(\varepsilon)}\right]^{\frac{N}{8}} k^{\frac{12-N}{4}}\left\|d_{t} \Theta_{\varphi}^{m}\right\|_{L^{2}}^{\frac{12-N}{4}} \\
& \left.+\left\|\Theta_{\varphi}^{m}\right\|_{L^{3}}^{3}\right\} \\
\leq & C\left\{\left\|\Theta_{\varphi}^{m-1}\right\|_{L^{2}}^{\frac{12-N}{4}} \mathcal{B}_{2}^{\frac{N}{8}}+\left\|\Theta_{\varphi}^{m}\right\|_{L^{3}}^{3}+\left\|\Upsilon_{\varphi}^{m-1}\right\|_{L^{2}}^{\frac{12-N}{4}} \mathcal{B}_{2}^{\frac{N}{8}}\right. \\
& \left.+C\left[\mathcal{B}_{2}+\frac{\mathcal{B}_{1}}{\alpha(\varepsilon)}\right]^{\frac{N}{8}} k^{2}\left(\left\|\Upsilon_{\varphi}^{m}\right\|_{L^{2}}+\left\|\Upsilon_{\varphi}^{m-1}\right\|_{L^{2}}\right)^{\frac{4-N}{4}}\left\|d_{t} \Upsilon_{\varphi}^{m}\right\|_{L^{2}}^{2}\right\} \\
& +C\left[\mathcal{B}_{2}+\frac{\mathcal{B}_{1}}{\alpha(\varepsilon)}\right]^{\frac{N}{8}}\left[\left\|\Theta_{\varphi}^{m}\right\|_{L^{2}}^{\frac{12-N}{4}}+\left\|\Theta_{\varphi}^{m-1}\right\|_{L^{2}}^{\frac{12-N}{4}}\right] .
\end{aligned}
$$

Summing over $m$ from 1 to $\ell$ and using (3.5)-(3.8), Lemmas 2.2 and 3.1, we get

$$
\begin{aligned}
\frac{k}{\varepsilon} \sum_{m=1}^{\ell} \| & \Upsilon_{\varphi}^{m} \|_{L^{3}}^{3} \\
\leq & C \frac{\mathcal{B}_{2}^{\frac{3}{2}}}{\varepsilon}\left\{1+\left[\mathcal{B}_{2}+\frac{\mathcal{B}_{1}}{\alpha(\varepsilon)}\right]^{\frac{N}{8}} \mathcal{B}_{2}^{-\frac{N}{8}}\right\} h^{\frac{12-N}{2}} \\
& +C \frac{\mathcal{B}_{2}^{\frac{N}{8}} k}{\varepsilon} \sum_{m=1}^{\ell}\left\|\Upsilon_{\varphi}^{m-1}\right\|_{L^{2}}^{\frac{12-N}{4}} \\
& +\frac{C}{\varepsilon}\left[\mathcal{B}_{2}+\frac{\mathcal{B}_{1}}{\alpha(\varepsilon)}\right]^{\frac{N}{8}}\left[\alpha(\varepsilon) \mathcal{J}_{\varepsilon}\left(\varphi_{0}^{\varepsilon}, u_{0}^{\varepsilon}\right)\right]^{\frac{4-N}{8}} k^{3} \sum_{m=1}^{\ell}\left\|d_{t} \Upsilon_{\varphi}^{m}\right\|_{L^{2}}^{2} .
\end{aligned}
$$

The last term of (3.42) can be absorbed by the corresponding term on the left-hand side of (3.37) if $k$ satisfies

$$
\frac{C}{\varepsilon}\left[\mathcal{B}_{2}+\frac{\mathcal{B}_{1}}{\alpha(\varepsilon)}\right]^{\frac{N}{8}}\left[\alpha(\varepsilon) \mathcal{J}_{\varepsilon}\left(\varphi_{0}^{\varepsilon}, u_{0}^{\varepsilon}\right)\right]^{\frac{4-N}{8}} k \leq \frac{\varepsilon \alpha(\varepsilon)}{8} .
$$

Hence, it follows from (3.37), (3.42) and (3.43) that

$$
\begin{aligned}
\frac{\varepsilon \alpha(\varepsilon)}{2}\left\|\Upsilon_{\varphi}^{\ell}\right\|_{L^{2}}^{2}+\frac{s(\varepsilon)}{2}\left\|\nabla G_{u}^{\ell}\right\|_{L^{2}}^{2}+k \sum_{m=1}^{\ell}\left\{\frac{\varepsilon \alpha(\varepsilon) k}{2}\left\|d_{t} \Upsilon_{\varphi}^{m}\right\|_{L^{2}}^{2}\right. \\
+\frac{s(\varepsilon) k}{2}\left\|\nabla \Upsilon_{u}^{m}\right\|_{L^{2}}^{2}+\frac{1}{\varepsilon}\left\|\Upsilon_{\varphi}^{m}\right\|_{L^{4}}^{4}+\frac{\varepsilon^{3} \alpha(\varepsilon)}{4}\left\|\nabla \Upsilon_{\varphi}^{m}\right\|_{L^{2}}^{2} \\
\left.+\frac{\varepsilon^{2} \alpha(\varepsilon) s(\varepsilon)}{4 c(\varepsilon)}\left\|\Upsilon_{\varphi}^{m}-\Delta_{h} G_{u}^{m}\right\|_{L^{2}}^{2}\right\} \\
\leq C\left[\eta(\varepsilon) h^{4}+\zeta(\varepsilon) k^{2}\right]+\left(C+8 C_{0}\right)\left\{\varepsilon \alpha(\varepsilon) k \sum_{m=0}^{\ell}\left\|\Upsilon_{\varphi}^{m}\right\|_{L^{2}}^{2}\right. \\
\left.+s(\varepsilon) k \sum_{m=0}^{\ell}\left\|\nabla G_{u}^{m}\right\|_{L^{2}}^{2}\right\}+\frac{C \mathcal{B}_{2}^{\frac{N}{8}}}{\varepsilon} k \sum_{m=0}^{\ell}\left\|\Upsilon_{\varphi}^{m-1}\right\|_{L^{2}}^{\frac{12-N}{4}}
\end{aligned}
$$

We note that the super-quadratic power in the last term allows us to control this error contribution by an inductive argument. 
Step 4. The details of the induction argument can be found in the proof of Theorem 3.1 of [26]. Here we only point out that this time the inductive argument leads to

$$
\begin{gathered}
\max _{0 \leq m \leq \ell}\left\{\frac{\varepsilon \alpha(\varepsilon)}{2}\left\|\Upsilon_{\varphi}^{m}\right\|_{L^{2}}^{2}+\frac{s(\varepsilon)}{2}\left\|\nabla G_{u}^{\ell}\right\|_{L^{2}}^{2}\right\}+k \sum_{m=1}^{\ell}\left\{\frac{\varepsilon \alpha(\varepsilon) k}{2}\left\|d_{t} \Upsilon_{\varphi}^{m}\right\|_{L^{2}}^{2}\right. \\
+\frac{s(\varepsilon) k}{2}\left\|\nabla \Upsilon_{u}^{m}\right\|_{L^{2}}^{2}+\frac{1}{\varepsilon}\left\|\Upsilon_{\varphi}^{m}\right\|_{L^{4}}^{4}+\frac{\varepsilon^{3} \alpha(\varepsilon)}{4}\left\|\nabla \Upsilon_{\varphi}^{m}\right\|_{L^{2}}^{2} \\
\left.+\frac{\varepsilon^{2} \alpha(\varepsilon) s(\varepsilon)}{4 c(\varepsilon)}\left\|\Upsilon_{\varphi}^{m}-\Delta_{h} G_{u}^{m}\right\|_{L^{2}}^{2}\right\} \\
\leq C\left[\eta(\varepsilon) h^{4}+\zeta(\varepsilon) k^{2}\right],
\end{gathered}
$$

provided that

$$
\eta(\varepsilon) h^{4}+\zeta(\varepsilon) k^{2} \leq \varepsilon^{-1}[\alpha(\varepsilon)]^{\frac{N-12}{4-N}} \mathcal{B}_{2}^{-\frac{N}{4-N}} .
$$

Finally, the assertions (i)-(iv) follow from (3.45, 3.5, 3.7) and applying the triangle inequality to $E_{u}^{m}=\Theta_{u}^{m}+\Upsilon_{u}^{m}$ and $E_{\varphi}^{m}=\Theta_{\varphi}^{m}+\Upsilon_{\varphi}^{m}$. The assertion (v) follows from applying the inverse inequality bounding the $L^{\infty}$ norm in terms of the $L^{2}$ norm, using 3.45 and 3.6 .

Remark 3.1. (a) The estimates in (i)-(iv) are optimal in both $h$ and $k$, and the one in (v) is quasi-optimal.

(b) The proof clearly shows how the three mesh conditions arise. Condition (1) is for the stability of the fully discrete scheme; condition (2) is required for having the discrete spectrum estimate (see Lemma 3.3); finally condition (3) is caused by the super-quadratic nonlinearity of $f$ (see Step 3 of the proof).

(c) Clearly, the $L^{2}$-projections $\Phi^{0}=Q_{h} \varphi_{0}^{\varepsilon}$ and $U^{0}=Q_{h} u_{0}^{\varepsilon}$ are valid choices for the starting values.

Theorem 3.2. Under the assumptions and mesh constraints of Theorem 3.1, there exists $h_{0}>0$ and $k_{0}>0$ (or there exists $\varepsilon_{1}>0$ ) such that the following error estimates hold for $h<h_{0}$ and $k<k_{0}$ (or for $\varepsilon<\varepsilon_{1}$ )

$$
\max _{0 \leq m \leq M}\left\|\Phi^{m}\right\|_{L^{\infty}} \leq 2 C_{0}
$$

$$
\begin{aligned}
& \max _{0 \leq m \leq M} \sqrt{\varepsilon c(\varepsilon) \alpha(\varepsilon)}\left\|u\left(t_{m}\right)-U^{m}\right\|_{L^{2}} \leq C\left[\delta_{1}(\varepsilon)^{\frac{1}{2}} h^{2}+\hat{\hat{\eta}}(\varepsilon)^{\frac{1}{2}} h^{2}+\hat{\hat{\zeta}}(\varepsilon)^{\frac{1}{2}} k\right], \\
& \sqrt{\varepsilon c(\varepsilon) s(\varepsilon)} \max _{0 \leq m \leq M}\left\|u\left(t_{m}\right)-U^{m}\right\|_{L^{\infty}}
\end{aligned}
$$

$$
\leq C\left\{\delta_{1}(\varepsilon)^{\frac{1}{2}} h^{\frac{4-N}{2}}|\ln h|^{\frac{3-N}{2}}+\left[\hat{\hat{\eta}}(\varepsilon)^{\frac{1}{2}} h^{2}+\hat{\hat{\zeta}}(\varepsilon)^{\frac{1}{2}} k\right] h^{-\frac{N}{2}}\right\},
$$

(v) $\left\{\varepsilon \alpha(\varepsilon) k \sum_{m=1}^{M}\left\|d_{t}\left(\varphi\left(t_{m}\right)-\Phi^{m}\right)\right\|_{L^{2}}\right\}^{\frac{1}{2}} \leq C\left[\delta_{3}(\varepsilon)^{\frac{1}{2}} h^{2}+\hat{\hat{\eta}}(\varepsilon)^{\frac{1}{2}} h^{2}+\hat{\hat{\zeta}}(\varepsilon)^{\frac{1}{2}} k\right]$,

$$
\begin{aligned}
& \left\{\varepsilon k \sum_{m=1}^{M} k\left\|d_{t} \nabla\left(\varphi\left(t_{m}\right)-\Phi^{m}\right)\right\|_{L^{2}}^{2}\right\}^{\frac{1}{2}} \leq C\left[\delta_{4}(\varepsilon)^{\frac{1}{2}} h+\hat{\hat{\eta}}(\varepsilon)^{\frac{1}{2}} h^{2}+\hat{\hat{\zeta}}(\varepsilon)^{\frac{1}{2}} k\right] \\
& \max _{0 \leq m \leq M}\left\|\nabla\left(u\left(t_{m}\right)-U^{m}\right)\right\|_{L^{2}} \leq C\left[\delta_{5}(\varepsilon)^{\frac{1}{2}} h+\hat{\hat{\eta}}(\varepsilon)^{\frac{1}{2}} h^{2}+\hat{\hat{\zeta}}(\varepsilon)^{\frac{1}{2}} k\right]
\end{aligned}
$$




$$
\begin{aligned}
& \text { (viii) } \quad\left\{c(\varepsilon) k \sum_{m=1}^{M}\left\|d_{t}\left(u\left(t_{m}\right)-U^{m}\right)\right\|_{L^{2}}\right\}^{\frac{1}{2}} \leq C\left[\delta_{6}(\varepsilon)^{\frac{1}{2}} h^{2}+\hat{\hat{\eta}}(\varepsilon)^{\frac{1}{2}} h^{2}+\hat{\hat{\zeta}}(\varepsilon)^{\frac{1}{2}} k\right], \\
& \text { (ix) } \quad\left\{k \sum_{m=1}^{M} k\left\|d_{t} \nabla\left(u\left(t_{m}\right)-U^{m}\right)\right\|_{L^{2}}^{2}\right\}^{\frac{1}{2}} \leq C\left[\delta_{7}(\varepsilon)^{\frac{1}{2}} h+\hat{\hat{\eta}}(\varepsilon)^{\frac{1}{2}} h^{2}+\hat{\hat{\zeta}}(\varepsilon)^{\frac{1}{2}} k\right],
\end{aligned}
$$

where

$$
\begin{aligned}
& \delta_{1}(\varepsilon)=\varepsilon c(\varepsilon) \alpha(\varepsilon) \mathcal{B}_{5}, \quad \delta_{2}(\varepsilon)=\varepsilon \mathcal{B}_{2}, \quad \delta_{3}(\varepsilon)=\frac{\alpha(\varepsilon)^{2} \mathcal{B}_{7}}{\varepsilon} \\
& \delta_{4}(\varepsilon)=\frac{\alpha(\varepsilon) k \mathcal{B}_{7}}{\varepsilon}, \quad \delta_{5}(\varepsilon)=\mathcal{B}_{5}, \quad \delta_{6}(\varepsilon)=\frac{c(\varepsilon) \mathcal{B}_{8}}{1+c(\varepsilon)}, \quad \delta_{7}(\varepsilon)=\frac{k \mathcal{B}_{8}}{1+c(\varepsilon)} \\
& \hat{\hat{\eta}}(\varepsilon)=\frac{\alpha(\varepsilon)^{2}}{\varepsilon} \mathcal{B}_{7}+\frac{s(\varepsilon)^{2}\left[\rho_{2}(\varepsilon)+\eta(\varepsilon)\right]}{\varepsilon c(\varepsilon) \alpha(\varepsilon)^{2}}+\frac{\rho_{1}(\varepsilon)+\eta(\varepsilon)}{\varepsilon^{3} \alpha(\varepsilon)} \\
& \hat{\hat{\zeta}}(\varepsilon)=\varepsilon \alpha(\varepsilon) \mathcal{B}_{7}+\frac{\mathcal{J}_{\varepsilon}\left(\varphi_{0}^{\varepsilon}, u_{0}^{\varepsilon}\right)+[1+c(\varepsilon)] \varepsilon^{-2 \sigma_{3}}}{c(\varepsilon)^{2}}+\frac{\zeta(\varepsilon)}{\varepsilon^{3} \alpha(\varepsilon)^{2}}+\frac{s(\varepsilon)^{2} \zeta(\varepsilon)}{\varepsilon c(\varepsilon) \alpha(\varepsilon)^{2}}
\end{aligned}
$$

Proof. The estimate (v) of Theorem 3.1 implies that there exist $h_{0}>0$ and $k_{0}>0$ (equivalently, there exists $\varepsilon_{1}>0$ ), such that

$$
\max _{0 \leq m \leq M}\left\|\varphi\left(t_{m}\right)-\Phi^{m}\right\|_{L^{\infty}} \leq \frac{C_{0}}{2}
$$

for $h<h_{0}$ and $k<k_{0}$ (or $\varepsilon<\varepsilon_{1}$ ), where $C_{0}>0$ is defined in Lemma 2.1. The assertion (i) then follows immediately from (2.5) and (3.49).

To show assertion (ii), taking $\eta_{h}=-\Upsilon_{u}^{m}$ and $v_{h}=\varepsilon \alpha(\varepsilon) \Upsilon_{u}^{m}$ in (3.21) and (3.22), respectively, and adding the resulting equations yields

$$
\begin{aligned}
& \frac{\varepsilon c(\varepsilon) \alpha(\varepsilon)}{2}\left[d_{t}\left\|\Upsilon_{u}^{m}\right\|_{L^{2}}^{2}+k\left\|d_{t} \Upsilon_{u}^{m}\right\|_{L^{2}}^{2}\right]+\varepsilon \alpha(\varepsilon)\left\|\nabla \Upsilon_{u}^{m}\right\|_{L^{2}}^{2} \\
& \quad=\varepsilon \alpha(\varepsilon) c(\varepsilon)\left[\left(\mathcal{R}_{u}^{m}, \Upsilon_{u}^{m}\right)-\left(d_{t} \Theta_{u}^{m}, \Upsilon_{u}^{m}\right)\right]+\varepsilon\left(\nabla \Upsilon_{\varphi}^{m}, \nabla \Upsilon_{u}^{m}\right) \\
& \quad-s(\varepsilon)\left(E_{u}^{m-1}, \Upsilon_{u}^{m}\right)-s(\varepsilon) k\left(d_{t} u\left(t_{m}\right), \Upsilon_{u}^{m}\right)-\frac{1}{\varepsilon}\left(f\left(\varphi\left(t_{m}\right)\right)-f\left(\Phi^{m}\right), \Upsilon_{u}^{m}\right)
\end{aligned}
$$

Summing the equation over $m$ from 1 to $\ell$ 's $(\leq M)$, then using Schwarz's inequality, (2.5), assertion (i) above, (i)-(iv) of Theorem[3.1] and (3.24) to bound the right-hand side, we get

$$
\begin{aligned}
\frac{\varepsilon c(\varepsilon) \alpha(\varepsilon)}{2}\left\|\Upsilon_{u}^{\ell}\right\|_{L^{2}}^{2} & +k \sum_{m=1}^{M}\left[\frac{\varepsilon c(\varepsilon) \alpha(\varepsilon) k}{2}\left\|d_{t} \Upsilon_{u}^{m}\right\|_{L^{2}}^{2}+\frac{\varepsilon \alpha(\varepsilon)}{2}\left\|\nabla \Upsilon_{u}^{m}\right\|_{L^{2}}^{2}\right] \\
& \leq C\left[\hat{\eta}(\varepsilon) h^{4}+\hat{\zeta}(\varepsilon) k^{2}\right]+\varepsilon c(\varepsilon) \alpha(\varepsilon) k \sum_{m=1}^{M}\left\|\Upsilon_{u}^{m}\right\|_{L^{2}}^{2}
\end{aligned}
$$

where $\hat{\zeta}(\varepsilon)$ and $\hat{\eta}(\varepsilon)$ are defined by (3.47) and (3.48), respectively.

Assertion (ii) follows from (3.5), (3.7) and (3.8), after applying Gronwall's inequality to (3.51). Assertion (iii) is obtained by using the estimate (ii), (3.6) and the inverse inequality bounding the $L^{\infty}$ norm in terms of the $L^{2}$ norm. 
To show assertions (iv)-(vi), we set $\eta_{h}=d_{t} \Upsilon_{\varphi}^{m}$ in (3.21) to get

$$
\begin{aligned}
\frac{\varepsilon \alpha(\varepsilon)}{2} \| d_{t} \Upsilon_{\varphi}^{m} & \left\|_{L^{2}}^{2}+\frac{\varepsilon}{2} d_{t}\right\| \nabla \Upsilon_{\varphi}^{m}\left\|_{L^{2}}^{2}+\frac{\varepsilon k}{2}\right\| d_{t} \nabla \Upsilon_{\varphi}^{m} \|_{L^{2}}^{2} \\
=\varepsilon \alpha(\varepsilon) & {\left[\left(\mathcal{R}_{\varphi}^{m}, d_{t} \Upsilon_{\varphi}^{m}\right)-\left(d_{t} \Theta_{\varphi}^{m}, d_{t} \Upsilon_{\varphi}^{m}\right)\right]+s(\varepsilon)\left(\Theta_{u}^{m-1}+\Upsilon_{u}^{m}, d_{t} \Upsilon_{\varphi}^{m}\right) } \\
& +s(\varepsilon) k\left[\left(d_{t} u\left(t_{m}\right), d_{t} \Upsilon_{\varphi}^{m}\right)+\left(d_{t} \Upsilon_{u}^{m}, d_{t} \Upsilon_{\varphi}^{m}\right)\right] \\
+ & \frac{1}{\varepsilon}\left(f\left(\varphi\left(t_{m}\right)\right)-f\left(\Upsilon_{\varphi}^{m}\right), d_{t} \Upsilon_{\varphi}^{m}\right) .
\end{aligned}
$$

Taking the sum over $m$ from 1 to $\ell(\leq M)$, using Schwarz's inequality, (i) and (ii) of Theorem 3.1 and (3.24) to bound the terms on the right-hand side and then applying Gronwall's inequality, we get

$$
\begin{aligned}
\max _{0 \leq m \leq \ell} \frac{\varepsilon}{2}\left\|\nabla \Upsilon_{\varphi}^{m}\right\|_{L^{2}}^{2} & +k \sum_{m=1}^{\ell}\left[\frac{\varepsilon \alpha(\varepsilon)}{4}\left\|d_{t} \Upsilon_{\varphi}^{m}\right\|_{L^{2}}^{2}+\frac{\varepsilon k}{2}\left\|d_{t} \nabla \Upsilon_{\varphi}^{m}\right\|_{L^{2}}^{2}\right] \\
& \leq C\left[\hat{\hat{\eta}}(\varepsilon) h^{4}+\hat{\hat{\zeta}}(\varepsilon) k^{2}\right],
\end{aligned}
$$

where $\hat{\hat{\eta}}(\varepsilon)$ and $\hat{\hat{\zeta}}(\varepsilon)$ are given by (3.47) and (3.48). Assertions (iv) $-($ vi) follow from applying the triangle inequality to $E_{\varphi}^{m}=\Theta_{\varphi}^{m}+\Upsilon_{\varphi}^{m}$ and using (3.5), (3.7) and (3.53).

Finally, setting $v_{h}=d_{t} \Upsilon_{u}^{m}$ in the error equation (3.22) gives

$$
\begin{aligned}
& \frac{c(\varepsilon)}{2}\left\|d_{t} \Upsilon_{u}^{m}\right\|_{L^{2}}^{2}+\frac{1}{2}\left[d_{t}\left\|\nabla \Upsilon_{u}^{m}\right\|_{L^{2}}^{2}+k\left\|d_{t} \nabla \Upsilon_{u}^{m}\right\|_{L^{2}}^{2}\right] \\
& \quad \leq C\left\{\frac{1}{c(\varepsilon)}\left[\left\|d_{t} E_{\varphi}^{m}\right\|_{L^{2}}^{2}+\left\|\mathcal{R}_{\varphi}^{m}\right\|_{L^{2}}^{2}\right]+c(\varepsilon)\left[\left\|d_{t} \Theta_{u}^{m}\right\|_{L^{2}}^{2}+\left\|\mathcal{R}_{u}^{m}\right\|_{L^{2}}^{2}\right]\right\}
\end{aligned}
$$

Then assertions (vii)-(ix) follow immediately from (3.54), (3.7), assertion (v) above, (3.24) and Gronwall's inequality.

\section{Convergence of Fully discrete solutions TO THE SOLUTIONS OF THE FREE BOUNDARY PROBLEMS}

In this section we shall present a nontrivial byproduct of the error estimates of the previous section. That is, we shall show convergence of the tuple $\left\{\left(\Phi^{m}, U^{m}\right)\right\}_{m=0}^{M}$ which solves the fully discrete mixed finite element scheme (3.1)-(3.2) to the solution of the free boundary problem (1.7)-(1.12), provided that the latter has a global (in time) classical solution. Specifically, it is proved that the fully discrete solution $U^{m}$, as $h, k \searrow 0$, converges to the solution $u^{0}$ of the phase field model uniformly in $\bar{\Omega}_{T}$, and the fully discrete solution $\Phi^{m}$ converges to \pm 1 uniformly on every compact subset of $\bar{\Omega}_{T} \backslash \Gamma$. Hence, the zero level set of $\Phi^{m}$ converges to the free boundary $\Gamma$. Our main ideas are to make full use of the convergence result that the free boundary problem is the distinguished limit, as $\varepsilon \searrow 0$, of the phase field model proved by Caginalp and Chen in [11, and to exploit the "closeness" between the solution $(\varphi, u)$ of the phase field model and its fully discrete approximation $\left\{\left(\Phi^{m}, U^{m}\right)\right\}_{m=0}^{M}$, which was obtained in the previous section. We note that as in [11], our numerical convergence is also established under the assumption that the free boundary problem has a global (in time) classical solution; we refer to [11 and references therein for further expositions on this assumption and related theoretical works on the phase field model. We also remark that our convergence result covers all six types of free boundary problems corresponding to six different sets of choices of $c^{0}, d^{0}$ and $\alpha^{0}$ in (1.7)-(1.12). These six types of free boundary problems include 
the classical Stefan problem, generalized Stefan problems with surface tension and surface kinetics, the Hele-Shaw problem, and the motion by mean curvature flow (cf. Theorem 4.1 below).

Let $\left(\varphi^{\varepsilon}, u^{\varepsilon}\right)$ denote the solution of the phase field model (1.1)-1.4). Note that we put back the super-index $\varepsilon$ on the solution in this section. Define $\left(\Phi_{\varepsilon, h, k}(x, t)\right.$, $\left.U_{\varepsilon, h, k}(x, t)\right)$ to be the piecewise linear interpolation (in time) of the fully discrete solution $\left(\Phi^{m}, U^{m}\right)$, that is,

$$
\begin{aligned}
\Phi_{\varepsilon, h, k}(\cdot, t) & :=\frac{t-t_{m}}{k} \Phi^{m+1}(\cdot)+\frac{t_{m+1}-t}{k} \Phi^{m}(\cdot), \\
U_{\varepsilon, h, k}(\cdot, t) & :=\frac{t-t_{m}}{k} U^{m+1}(\cdot)+\frac{t_{m+1}-t}{k} U^{m}(\cdot)
\end{aligned}
$$

for $t_{m} \leq t \leq t_{m+1}$ and $0 \leq m \leq M-1$. Note that $\Phi_{\varepsilon, h, k}$ and $U_{\varepsilon, h, k}$ are continuous piecewise linear functions in space and time.

Let $\Gamma_{00} \subset \Omega$ be a smooth closed hypersurface and let $\left(u^{0}, \Gamma:=\bigcup_{0 \leq t \leq T}\left(\Gamma_{t} \times\{t\}\right)\right)$ be a smooth solution of the free boundary problem (1.7)-(1.12) starting from $\Gamma_{00}$ such that $\Gamma \subset \Omega \times[0, T]$. Let $d(x, t)$ denote the signed distance function to $\Gamma_{t}$ such that $d(x, t)<0$ in $\mathcal{I}_{t}$, the inside of $\Gamma_{t}$, and $d(x, t)>0$ in $\mathcal{O}_{t}:=\Omega \backslash\left(\Gamma_{t} \cup \mathcal{I}_{t}\right)$, the outside of $\Gamma_{t}$. We also define the inside $\mathcal{I}$ and the outside $\mathcal{O}$ of $\Gamma$ as follows:

$$
\begin{aligned}
& \mathcal{I}:=\{(x, t) \in \Omega \times[0, T] ; d(x, t)<0\}, \\
& \mathcal{O}:=\{(x, t) \in \Omega \times[0, T] ; d(x, t)>0\} .
\end{aligned}
$$

In addition, let $\Gamma_{t}^{\varepsilon, h, k}$ denote the zero level set of $\Phi_{\varepsilon, h, k}$ at time $t$, that is,

$$
\Gamma_{t}^{\varepsilon, h, k}:=\left\{x \in \Omega ; \Phi_{\varepsilon, h, k}(x, t)=0\right\} .
$$

As mentioned earlier, our proof of convergence is based on making full use of the convergence result of [11, which shows that the free boundary problem is the distinguished limit, as $\varepsilon \searrow 0$, of the phase field model. For the readers' convenience, we recall the convergence result in the following theorem, and we refer to Theorems 2.1 and 2.2 of [11] for more details.

Theorem 4.1. Let $\Omega$ be a given smooth domain and let $\Gamma_{00}$ be a smooth closed hypersurface in $\Omega$. Suppose that the free boundary problem (1.7)-1.12) starting from $\Gamma_{00}$ has a smooth solution $\left(u^{0}, \Gamma:=\bigcup_{0 \leq t \leq T}\left(\Gamma_{t} \times\{t\}\right)\right)$ such that $\Gamma \subset \Omega \times[0, T]$. Then there exists a family of smooth functions $\left\{\left(\varphi_{0}^{\varepsilon}, u_{0}^{\varepsilon}\right)\right\}_{0<\varepsilon \leq 1}$ which are uniformly bounded in $\varepsilon \in(0,1]$ and $(x, t) \in \bar{\Omega}_{T}$, such that if $\left(\varphi^{\varepsilon}, u^{\varepsilon}\right)$ solves the phase field model (1.1) $-(1.4)$, then

$$
\text { (i) } \quad\left\|u^{\varepsilon}-u^{0}\right\|_{C^{0}\left(\bar{\Omega}_{T}\right)} \stackrel{\varepsilon \backslash 0}{\longrightarrow} 0,
$$$$
\text { (ii) } \varphi^{\varepsilon} \stackrel{\varepsilon \searrow 0}{\longrightarrow} 1 \text { uniformly on compact subsets of } \mathcal{O} \text {, }
$$$$
\text { (iii) } \quad \varphi^{\varepsilon} \stackrel{\varepsilon \searrow 0}{\longrightarrow}-1 \quad \text { uniformly on compact subsets of } \mathcal{I}
$$

hold in each of the following six cases:

(1) $\alpha^{0}, c^{0}$ and $d_{0}$ are positive constants in (1.7)-(1.12); $\alpha(\varepsilon)=\alpha^{0}, c(\varepsilon)=$ $c^{0}$ and $s(\varepsilon)=\frac{m}{2 d_{0}}$ in (1.1)-(1.4), where $m=\int_{-1}^{1} \sqrt{2 F(s)} \mathrm{d} s$, which only depends on the choice of the potential function $F$.

(2) $d^{0}=0, \alpha^{0}$ and $c^{0}$ are positive constants; $\alpha(\varepsilon)=\alpha^{0}, c(\varepsilon)=c^{0}$ and $s(\varepsilon)=$ $\varepsilon^{-\frac{1}{2}}$. 
(3) $c^{0}=0, \alpha^{0}$ and $d^{0}$ are positive constants; $c(\varepsilon)=\varepsilon^{\ell}$ for any $\ell \geq 1, \alpha(\varepsilon)=\alpha^{0}$ and $s(\varepsilon)=\frac{m}{2 d_{0}}$.

(4) $c^{0}$ and $\alpha^{0}$ are positive constants; $\alpha(\varepsilon)=\alpha^{0}, c(\varepsilon)=c^{0}, s(\varepsilon)=\varepsilon^{\ell}$ for any $\ell \geq 1$. In this case, the equilibrium condition (1.10) is replaced by $\kappa_{\Gamma}=\alpha^{0} V$.

(5) $\alpha^{0}=0, d^{0}$ and $c^{0}$ are positive constants; and $\alpha(\varepsilon)=\varepsilon^{\ell}$ for any $\ell \geq 1$, $c(\varepsilon)=c^{0}$ and $s(\varepsilon)=\frac{m}{2 d_{0}}$.

(6) $c^{0}=\alpha^{0}=0, d^{0}$ is positive constant; $c(\varepsilon)=\varepsilon^{\ell}, \alpha(\varepsilon)=\varepsilon^{j}$ for any $\ell, j \geq 1$, and $s(\varepsilon)=\frac{m}{2 d_{0}}$.

Remark 4.1. The above convergence result was proved in 11 when $\varphi$ satisfies the Dirichlet boundary condition. However, it was noted in (4) of Remark 2.3 of [1] (see page 424 of [11) that the conclusion still holds when $\varphi$ satisfies the homogeneous Neumann boundary condition.

We also remark that the free boundary problem in case (2) is the classical Stefan problem. The free boundary condition (1.10) in case (5) reduces to the well-known Gibbs-Thomson condition. The free boundary problem in case (4) is the motion by mean curvature flow [20, 22, and the one in case (6) is known as the HeleShaw/Mullins-Sekerka problem 3, 33. The remaining cases (1) and (3) are the generalized Stefan problems with surface tension and surface kinetics.

We are now ready to show the following convergence theorem.

Theorem 4.2. Let $\Omega$ be a given smooth domain and let $\Gamma_{00}$ be a smooth closed hypersurface in $\Omega$. Suppose that the free boundary problem (1.7)-(1.12) starting from $\Gamma_{00}$ has a classical solution $\left(u^{0}, \Gamma:=\bigcup_{0 \leq t \leq T}\left(\Gamma_{t} \times\{t\}\right)\right)$ such that $\Gamma_{t} \subset \Omega$ for all $t \in[0, T]$. Let $\left\{\left(\varphi_{0}^{\varepsilon}, u_{0}^{\varepsilon}\right)\right\}_{0<\varepsilon \leq 1}$ be the family of smooth uniformly bounded functions as in Theorem 2.1 and 2.2 of [11. Let $\left(\Phi_{\varepsilon, h, k}(x, t), U_{\varepsilon, h, k}(x, t)\right)$ denote the piecewise linear interpolation (in time) of the fully discrete solution $\left\{\left(\Phi^{m}, U^{m}\right)\right\}_{m=0}^{M}$ of (3.1) - (3.2). Also, let $\mathcal{I}$ and $\mathcal{O}$ stand for the "inside" and "outside" (in $\Omega_{T}$ ) of $\Gamma$. Then under the mesh and starting value constraints of Theorem 3.1 we have

$$
\begin{aligned}
& \left\|U_{\varepsilon, h, k}-u^{0}\right\|_{C^{0}\left(\bar{\Omega}_{T}\right)} \stackrel{\varepsilon \searrow 0}{\longrightarrow} 0, \\
& \Phi_{\varepsilon, h, k}(x, t) \stackrel{\varepsilon \searrow 0}{\longrightarrow} 1 \quad \text { uniformly on compact subset of } \mathcal{O}, \\
& \Phi_{\varepsilon, h, k}(x, t) \stackrel{\varepsilon \searrow 0}{\longrightarrow}-1 \quad \text { uniformly on compact subset of } \mathcal{I}
\end{aligned}
$$

in each of the six cases of different combinations of $c^{0}, \alpha^{0}$ and $d^{0}$ as described in Theorem 4.1.

Proof. The triangle inequality implies that

$$
\left\|U_{\varepsilon, h, k}-u^{0}\right\|_{C^{0}\left(\bar{\Omega}_{T}\right)} \leq\left\|U_{\varepsilon, h, k}-u^{\varepsilon}\right\|_{C^{0}\left(\bar{\Omega}_{T}\right)}+\left\|u^{\varepsilon}-u^{0}\right\|_{C^{0}\left(\bar{\Omega}_{T}\right)} .
$$

Assertion (i) then follows immediately from (i) of Theorem 4.1 and the $L^{\infty}$ error estimate given in Theorem 3.2 .

To show assertion (ii), let $A$ be any compact subset of $\mathcal{O}$, for any $(x, t) \in A$, using the triangle inequality we have

$$
\begin{aligned}
\left|\Phi_{\varepsilon, h, k}(x, t)-1\right| & \leq\left|\Phi_{\varepsilon, h, k}(x, t)-\varphi^{\varepsilon}(x, t)\right|+\left|\varphi^{\varepsilon}(x, t)-1\right| \\
& \leq\left\|\Phi_{\varepsilon, h, k}-\varphi^{\varepsilon}\right\|_{L^{\infty}\left(\Omega_{T}\right)}+\left|\varphi^{\varepsilon}(x, t)-1\right| .
\end{aligned}
$$


Under the assumptions of Theorem 4.2 the first term on the right-hand side of (4.7) is bounded by $C\left(h^{\alpha}+k^{\beta}\right)$ for some $\alpha, \beta>0$; hence, it converges to zero uniformly on $A$ (and on $\Omega$ ) as $h \searrow 0$. From (ii) of Theorem 4.1 we know that the second term on the right-hand side of (4.7) also converges to zero uniformly on $A$. Note that $h \searrow 0$ as $\varepsilon \searrow 0$. Therefore,

$$
\Phi_{\varepsilon, h, k} \stackrel{\varepsilon \backslash 0}{\longrightarrow} 1 \text { uniformly on } A .
$$

This then completes the proof of assertion (ii).

The proof of assertion (iii) is almost a repetition of the above proof. The only change is to replace $\mathcal{O}$ by $\mathcal{I}$ and 1 by -1 in the above proof. So we omit it.

Another consequence of Theorems 3.1 and 3.2 is the following convergence result for the numerical interface, which establishes convergence of the zero level set $\Gamma_{t}^{\varepsilon, h, k}$ of $\Phi_{\varepsilon, h, k}$ to the true free boundary $\Gamma_{t}$.

Theorem 4.3. Let $\Gamma_{t}^{\varepsilon, h, k}:=\left\{x \in \Omega ; \Phi_{\varepsilon, h, k}(x, t)=0\right\}$ denote the zero level set of $\Phi_{\varepsilon, h, k}$. Then under the assumptions of Theorem 4.2, it holds that

$$
\sup _{x \in \Gamma_{t}^{\varepsilon, h, k}}\left(\operatorname{dist}\left(x, \Gamma_{t}\right)\right) \stackrel{\varepsilon \backslash 0}{\longrightarrow} 0 \quad \text { uniformly on }[0, T] \text {. }
$$

Proof. For any $\delta \in(0,1)$, define the (open) tubular neighborhood $\mathcal{N}_{\delta}$ of width $2 \delta$ of $\Gamma$ as

$$
\mathcal{N}_{\delta}:=\left\{(x, t) \in \Omega_{T} ; d(x, t)<\delta\right\} .
$$

Let $A$ and $B$ denote the complements of $\mathcal{N}_{\delta}$ in $\mathcal{O}$ and $\mathcal{I}$, respectively, that is

$$
A=\mathcal{O} \backslash \mathcal{N}_{\delta}, \quad B=\mathcal{I} \backslash \mathcal{N}_{\delta} .
$$

Note that $A$ is a compact subset of $\mathcal{O}$ and $B$ is a compact subset of $\mathcal{I}$. Hence, from (ii) and (iii) of Theorem 4.2 we know that there exists $\widehat{\varepsilon}_{0}>0$, which only depends on $\delta$, such that for all $\varepsilon \in\left(0, \widehat{\varepsilon}_{0}\right)$

$$
\begin{aligned}
& \left|\Phi_{\varepsilon, h, k}(x, t)-1\right| \leq \delta \quad \forall(x, t) \in A, \\
& \left|\Phi_{\varepsilon, h, k}(x, t)+1\right| \leq \delta \quad \forall(x, t) \in B .
\end{aligned}
$$

Now for any $t \in[0, T]$ and $x \in \Gamma_{t}^{\varepsilon, h, k}$, since $\Phi_{\varepsilon, h, k}(x, t)=0$, we have

$$
\begin{aligned}
& \left|\Phi_{\varepsilon, h, k}(x, t)-1\right|=1, \\
& \left|\Phi_{\varepsilon, h, k}(x, t)+1\right|=1 .
\end{aligned}
$$

Evidently, (4.9) and (4.11) imply that $(x, t) \notin A$, and (4.10) and (4.12) say that $(x, t) \notin B$. Hence $(x, t)$ must reside in the tubular neighborhood $\mathcal{N}_{\delta}$. Since $t$ is an arbitrary number in $[0, T]$ and $x$ is an arbitrary point on $\Gamma_{t}^{\varepsilon, h, k}$, therefore, for all $\varepsilon \in\left(0, \widehat{\varepsilon}_{0}\right)$

$$
\sup _{x \in \Gamma_{t}^{\varepsilon, h, k}}\left(\operatorname{dist}\left(x, \Gamma_{t}\right)\right) \leq \delta \quad \text { uniformly on }[0, T] .
$$

The proof is complete.

Remark 4.2. Unlike as in 23], Theorem 4.3 does not provide any information about the rate of convergence of the numerical free boundary $\Gamma_{t}^{\varepsilon, h, k}$ to the true free boundary $\Gamma_{t}$. This is because no rate of convergence for the zero level set of $\varphi^{\varepsilon}$ to $\Gamma_{t}$ was obtained in [11]. To our knowledge, such a rate of convergence estimate is not known in the literature. On the other hand, if a rate of convergence can be proved 
for the zero level set of $\varphi^{\varepsilon}$ to the true free boundary $\Gamma_{t}$, it is easy to show that our numerical free boundary $\Gamma_{t}^{\varepsilon, h, k}$ should enjoy at least the same rate of convergence to the true free boundary $\Gamma_{t}$.

\section{ACKNOWLEDGMENTS}

The first author would like to thank Xinfu Chen for answering his questions about the spectrum estimates in Lemma 2.3. The second author gratefully acknowledges financial support by the Deutsche Forschungsgemeinschaft. Both authors would like to thank the referee for carefully reading the paper and for providing some valuable suggestions to improve the paper.

\section{REFERENCES}

1. R. A. Adams. Sobolev Spaces. Academic Press, New York, 1975. MR 56:9247

2. N. D. Alikakos and P. W. Bates. On the singular limit in a phase field model of phase transitions. Ann. Inst. H. Poincaré Anal. Non Linéaire, 5(2):141-178, 1988. MR 89h:35109

3. N. D. Alikakos, P. W. Bates, and X. Chen. Convergence of the Cahn-Hilliard equation to the Hele-Shaw model. Arch. Rational Mech. Anal., 128(2):165-205, 1994. MR 97b:35174

4. S. Allen and J. W. Cahn. A microscopic theory for antiphase boundary motion and its application to antiphase domain coarsening. Acta Metall., 27:1084-1095, 1979.

5. D. M. Anderson, G. B. McFadden, and A. A. Wheeler. A phase-field model of solidification with convection. Phys. D, 135(1-2):175-194, 2000. MR 2000j:80004

6. P. W. Bates, P. C. Fife, R. A. Gardner, and C. K. R. T. Jones. Phase field models for hypercooled solidification. Phys. D, 104(1):1-31, 1997. MR 97m:80011

7. J. F. Blowey and C. M. Elliott. A phase-field model with a double obstacle potential. In G. Buttazzo and A. Visintin, editors, Motion by mean curvature and related topics (Trento, 1992), pages 1-22. de Gruyter, Berlin, 1994. MR 95d:35093

8. S. C. Brenner and L. R. Scott. The mathematical theory of finite element methods. SpringerVerlag, New York, 1994. MR 95f:65001

9. G. Caginalp. An analysis of a phase field model of a free boundary. Arch. Rational Mech. Anal., 92(3):205-245, 1986. MR 87c:80011

10. G. Caginalp. Stefan and Hele-Shaw type models as asymptotic limits of the phase-field equations. Phys. Rev. A (3), 39(11):5887-5896, 1989. MR 90c:80004

11. G. Caginalp and X. Chen. Convergence of the phase field model to its sharp interface limits. European J. Appl. Math., 9(4):417-445, 1998. MR 99i:80011

12. G. Caginalp and J.-T. Lin. A numerical analysis of an anisotropic phase field model. IMA J. Appl. Math., 39(1):51-66, 1987. MR 90e:80005

13. G. Caginalp and E. Socolovsky. Phase field computations of single-needle crystals, crystal growth, and motion by mean curvature. SIAM J. Sci. Comput., 15(1):106-126, 1994. MR 94k:65122

14. J. W. Cahn and J. E. Hilliard. Free energy of a nonuniform system I. Interfacial free energy. J. Chem. Phys., 28:258-267, 1958.

15. X. Chen. Spectrum for the Allen-Cahn, Cahn-Hilliard, and phase-field equations for generic interfaces. Comm. Partial Differential Equations, 19(7-8):1371-1395, 1994. MR 95f:35023

16. X. Chen, J. Hong, and F. Yi. Existence, uniqueness, and regularity of classical solutions of the Mullins-Sekerka problem. Comm. Partial Differential Equations, 21(11-12):1705-1727, 1996. MR 97h:35238

17. Z. M. Chen and K.-H. Hoffmann. An error estimate for a finite-element scheme for a phase field model. IMA J. Numer. Anal., 14(2):243-255, 1994. MR 95c:65152

18. P. G. Ciarlet. The finite element method for elliptic problems. North-Holland Publishing Co., Amsterdam, 1978. Studies in Mathematics and its Applications, Vol. 4. MR 58:25001

19. J. B. Collins and H. Levine. Diffuse interface model of diffusion-limited crystal growth. Phys. Rev. B, 31:6119-6122, 1985.

20. P. de Mottoni and M. Schatzman. Geometrical evolution of developed interfaces. Trans. Amer. Math. Soc., 347(5):1533-1589, 1995. MR 2000a:35022 
21. C. M. Elliott and S. M. Zheng. Global existence and stability of solutions to the phase field equations. In Free Boundary Value Problems (Oberwolfach, 1989), pages 46-58. Birkhäuser, Basel, 1990. MR 92g:35214

22. L. C. Evans, H. M. Soner, and P. E. Souganidis. Phase transitions and generalized motion by mean curvature. Comm. Pure Appl. Math., 45(9):1097-1123, 1992. MR 93g:35064

23. X. Feng and A. Prohl. Numerical analysis of the Allen-Cahn equation and approximation of the mean curvature flows. Numer. Math., 94(1):33-65, 2003.

24. X. Feng and A. Prohl. Error Analysis of a Mixed Finite Element Method for the Cahn-Hilliard Equation, Numer. Math. (submitted), IMA-Preprint \#1798, 2001.

25. X. Feng and A. Prohl. Numerical Analysis of the Cahn-Hilliard Equation and Approximation for the Hele-Shaw problem, Interfaces and Free Boundaries (submitted), IMA-Preprint \#1799, 2001.

26. X. Feng and A. Prohl. Analysis of a fully discrete finite element method for the phase field model and approximation of its sharp interphase limits. IMA-Preprint \#1817, 2001.

27. P. Fife. Models for phase separation and their mathematics. Electronic J. of Diff. Eqns, 2000(48):1-26, 2000. MR 2001k:35147

28. G. Fix. Phase field method for free boundary problems. In A. Fasano and M. Primicerio, editors, Free Boundary Problems, pages 580-589. Pitman, London, 1983. MR 84h:00003

29. G. J. Fix and J. T. Lin. Numerical simulations of nonlinear phase transitions. I. The isotropic case. Nonlinear Anal., 12(8):811-823, 1988. MR 89h:80009

30. J. S. Langer. Models of patten formation in first-order phase transitions. In Directions in Condensed Matter Physics, pages 164-186. World Science Publishers, 1986. MR 88a:82023

31. J. T. Lin. The numerical analysis of a phase field model in moving boundary problems. SIAM J. Numer. Anal., 25(5):1015-1031, 1988. MR 89h:65161

32. G. B. McFadden, A. A. Wheeler, R. J. Braun, S. R. Coriell, and R. F. Sekerka. Phase-field models for anisotropic interfaces. Phys. Rev. E (3), 48(3):2016-2024, 1993.

33. W. W. Mullins and J. Sekerka. Morphological stability of a particle growing by diffusion or heat flow. J. Appl. Math., 34:322-329, 1963.

34. O. Penrose and P. C. Fife. On the relation between the standard phase-field model and a "thermodynamically consistent" phase-field model. Phys. D, 69(1-2):107-113, 1993.

35. N. Provatas, N. Goldenfeld, and J. Dantzig. Adaptive mesh refinement computation of solidification microstructures using dynamic data structures. J. Comput. Phys., 148(1):265-290, 1999. MR 99h:80012

36. H. M. Soner. Convergence of the phase-field equations to the Mullins-Sekerka problem with kinetic undercooling. Arch. Rational Mech. Anal., 131(2):139-197, 1995. MR 97d:80007

37. B. E. E. Stoth. A sharp interface limit of the phase field equations: one-dimensional and axisymmetric. European J. Appl. Math., 7(6):603-633, 1996. MR 98i:80008

38. X. Y. Yue. Finite element analysis of the phase field model with nonsmooth initial data. Acta Math. Appl. Sinica, 19(1):15-24, 1996. MR 97d:65045

Department of Mathematics, The University of Tennessee, Knoxville, Tennessee 37996

E-mail address: xfeng@math.utk.edu

Department of Mathematics, ETH, CH-8092 Zürich, Switzerland

E-mail address: apr@math.ethz.ch 